\title{
Variability of quantal synaptic currents in thalamocortical neurons
}

\author{
M. Neubig ${ }^{a, *}$, A. Destexhe ${ }^{\text {b }}$, T.J. Sejnowski ${ }^{\mathrm{c}}$

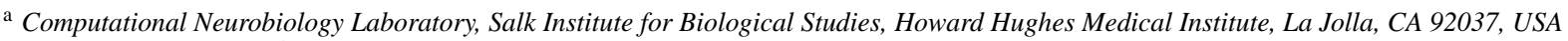 \\ b Integrative and Computational Neuroscience Unit (UNIC), CNRS, Gif-sur-Yvette, France \\ ${ }^{\mathrm{c}}$ Division of Biological Sciences, University of California at San Diego, La Jolla, CA 92093, USA
}

Accepted 17 January 2003

\begin{abstract}
Broad amplitude variability and skewed distributions are characteristic features of quantal synaptic currents (minis) at central synapses. The relative contributions of the various underlying sources are still debated. Through computational models of thalamocortical neurons, we separated intra- from extra-synaptic sources. Our simulations indicate that the external factors of local input resistance and dendritic filtering generate equally small amounts of negatively skewed synaptic variability. The ability of these two factors to reduce positive skew increased as their contribution to variability increased, which in control trials for morphological, biophysical, and experimental parameters never exceeded $10 \%$ of the range. With these dendritic factors ruled out, we tested multiple release models, which led to distributions with clearly non-physiological multiple peaks. We conclude that intra-synaptic organization is the primary determinant of synaptic variability in thalamocortical neurons and, due to extra-synaptic mechanisms, is more potent than the data suggested. Thalamortical neurons, especially in rodents, constitute a remarkably favorable system for molecular genetic studies of synaptic variability and its functional consequence. (C) 2003 Elsevier Science Ltd. All rights reserved.
\end{abstract}

Keywords: Computational models; Molecular genetics; Rodents; Minis; Dendrites

\section{Introduction}

In many neurons, quantal synaptic currents vary widely in peak amplitude. This amplitude variability is readily observed in somatic voltage clamp recordings following application of TTX, a sodium channel blocker that prevents action potential generation. Peak response amplitudes typically follow distributions that are broad, unimodal, and skewed towards low amplitude events (Edwards et al., 1990; Frerking et al., 1997; Stevens, 1993; Walmsley et al., 1998). This is true for large neurons (hippocampal pyramidal neurons (Brown et al., 1979; Ropert et al., 1990)), for compact neurons (retinal amacrine cells, cerebellar granule cells (Frerking et al., 1995; Silver et al., 1992)), and for bushy neurons (thalamocortical relay neurons (TCN) (Cox et al., 1997; LeFeuvre et al., 1997; Leresche, 1992; Ohara and Havton, 1994; Ohara et al., 1995; Ulrich and Huguenard, 1996; Zhang et al., 1997)).

Mechanisms that have been proposed to underly such distributions include intra-synaptic organization: (1) vesicle size/content, (2) re-uptake mechanisms, (3) cleft geometry, (4) receptor number, subtype, and modulation, and

\footnotetext{
* Corresponding author. Tel.: +1-858-453-4100x1463; fax: +1-858-587-0417.

E-mail address: neubig@salk.edu (M. Neubig).
}

extra-synaptic processing, (5) activation of voltage gated conductances, (6) resistive-capacitive filtering, (7) site dependent local input resistance, (8) site dependent space clamp quality, and (9) temporal overlap of events.

Consensus has not developed regarding the relative importance of these sources of variability. Resistive-capacitive filtering accounts for half the amplitude range in some neurons (Rall, 1967, 1977; Spruston et al., 1994; Stuart and Spruston, 1998; Smetters and Nelson, 1994, 1995), yet certain individual intra-synaptic factors account for the entire distribution in others (Faber et al., 1992; Frerking et al., 1995; Nusser et al., 1997, 1998).

That the range and skew of amplitude histograms might be important in brain function may be inferred from the work of Turrigiano et al., where it has been shown that synaptic weights scale multiplicatively, in inverse proportion to the long term $(48 \mathrm{~h})$ level of synaptic activity (Turrigiano et al., 1998). Multiplicative plasticity preserves skew, implying that the distribution's shape carries a functional significance at the level of networks.

Here, the pertinent network is comprised of reciprocal, collateral and intra-nuclear connections occurring between the neocortex, dorsal thalamus and ventral thalamus (Steriade et al., 1993). Other inputs include state dependent drives from the brainstem and basal forebrain (McCormick, 1992). 
Inhibition onto thalamocortical neurons has one of two network functions depending on the behavioral state of the animal (Andersen and Eccles, 1962; Andersen and Sears, 1964; Destexhe et al., 1996, 1998a,b; Sohal and Huguenard, 1998; Sohal et al., 2000). During quiet sleep, this $G A B A$-ergic activity is generic and phasic, while during wakefulness, the activity is singular and tonic. The activity elicits rebound burst firing (Coulter et al., 1989; Crunelli et al., 1989; Jahnsen and Llinás, 1984b) that is essential for corticothalamic oscillations (Steriade et al., 1993) during sleep. During wakefulness, it sculpts dendritic processing of ascending sensory information (Funke and Eysel, 1998; Hicks et al., 1986; Lee and Ebner, 1992; Lee et al., 1994; Salt, 1989; Worgotter et al., 1998).

In many species, this inhibitory drive stems from several structures in the network and arrives on dendritic spines. This is not the case in the rodent ventrobasal thalamus, where inhibition derives from a single structure-the thalamic reticular nucleus - and occurs directly on dendritic shafts at synapses located throughout the arbor (Benson et al., 1992; Harris et al., 1987; Jones, 1991; Liu et al., 1995; Ohara and Lieberman, 1993; Sato et al., 1997; Williams and Faull, 1987). Moreover, the thalamic reticular nucleus is comprised of single class of neurons, as is the rodent ventrobasal thalamus, which makes it an ideal system for studying synaptic variability. Using computational models of the relay neurons of this structure, we have explored sources of synaptic variability and have contrasted them with in vitro data.

\section{Method}

A biocytin filled thalamocortical relay neuron (TCN) of the ventrobasal complex of young rat was digitized with 13 somatic and 1198 dendritic data points using light mi- croscopy $(60-100 \times)$ and a computerized tracing system (Eutectic Electronics, Raleigh, NC). Distance between data points was $6.0 \pm 2.8 \mu \mathrm{m}(\mu \pm \sigma)$. This reconstruction (Fig. 1A) has been used in our earlier work, where details may be found (Destexhe et al., 1998a,b; Neubig, 1999; Neubig and Destexhe, 1997, 1998a,b, 1999, 2000, 2001).

During reconstruction, 27 fictional data points had been used to accommodate junctions with more than 2 daughter branches. These were removed and the $n$-tuple junctions re-established in the final model, which comprised 178 branches totaling $7092 \mu \mathrm{m}$ and $21366 \mu \mathrm{m}^{2}$ in length and area. Additional arbor statistics are presented in (Fig. 8).

Final somatic area $\left(2870 \mu \mathrm{m}^{2}\right)$ excluded the $301 \mu \mathrm{m}^{2}$ of fictional membrane circumscribed by the 11 dendritic trunks. Fictional area was calculated as $\pi \times\left[c \times R_{\text {trunk }}\right]^{2}$, where the factor $c=1.5$ was introduced to account for the curved transition between dendrite and soma. Trunks measured $R_{\text {trunk }}=$ $1.8 \pm 0.7 \mu \mathrm{m}$, range $0.8-2.7 \mu \mathrm{m}$.

Distal dendrites of rodent ventrobasal TCNs have uniform diameters (Ohara and Havton, 1994; Ohara et al., 1995). In the biocytin filled TCN, several places had periodic swellings. These were corrected by imposing a uniform diameter while conserving membrane area. To assess the corrections, simulations were run on morphological variants of the reconstructed arbor (Figs. 7 and 8). These simulations also served as controls for the slice, filling, fixation, and reconstruction processes, and established the pertinence of our conclusions to TCNs in general and to other neurons with similar dendritic architectures.

For numerical accuracy in the simulations, each branch was divided into $k$ compartments of length $l=L / k ; L$ is the anatomical length; $k$ the least integer such that $l / \lambda \leq \delta$

$$
\lambda=\sqrt{\left(\frac{1}{g \times R_{\mathrm{a}}} \times \frac{A}{\pi \times l} \times \frac{c}{4}\right)}
$$
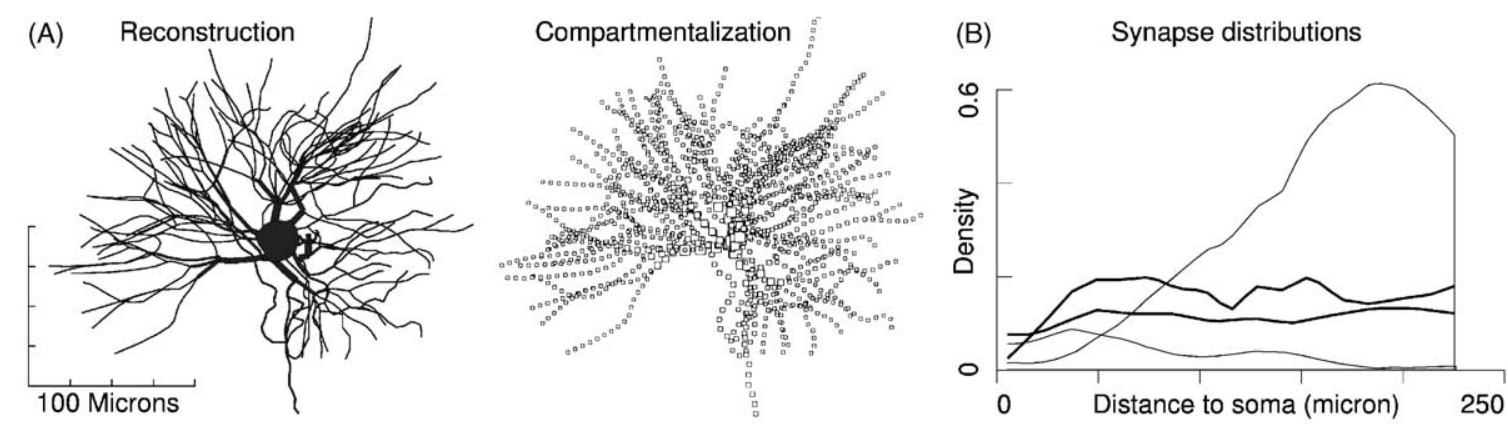

Fig. 1. Dendritic morphology and synapse distributions: (A) thalamocortical relay neuron (TCN) from the ventrobasal complex of the somatosensory thalamus of rat. Note the rapid reduction of dendrite diameter, long terminal branches and short primary and intermediate branches that are characteristic of TCNs and are defining characteristics of bushy neurons. Morphology is shown flattened to viewing plane (not projected). Additional morphology data appears in Fig. 8. Electrophysiological recordings from this neuron as well as its 3D reconstruction data and were used to create a computational model. The second panel depicts each compartment $(N=1416)$ of the model, with the areas of squares being proportional to compartment membrane area. (B) Uniform area density of inhibitory synapses (heavy lines; top (Sato et al., 1997), bottom (Sato et al., 1997; Liu et al., 1995)) contrasts with non-uniform densities of excitatory synapses from "cortical" (upper thin line) and "sensory" (lower thin line) afferents. Based on serial reconstructive electron microscopy data (Sato et al., 1997; Liu et al., 1995). Individual TCNs receive approximately 1500 inhibitory synapses, which, in rodent ventrobasal complex, derive from the reticular thalamic nucleus only. 
where $A$ is the compartment membrane area, $g$ the membrane conductance density, $R_{\mathrm{a}}$ the axial resistivity; and $c$ the conversion factor. Setting $g, R_{\mathrm{a}}, \delta$ to $39 \mu \mathrm{S} / \mathrm{cm}^{2}$, $200 \Omega \mathrm{cm}, 0.01 \lambda$ led to 1416 compartments of length $0.0094 \pm 0.0008 \lambda$, range $0.010-0.0019 \lambda(5.00 \pm 0.67 \mu \mathrm{m}$; range $1.00-11.02 \mu \mathrm{m}) . R_{\mathrm{a}}$ and $g$ had been obtained by fitting behavior to whole cell recordings from the same neuron (details in our previous studies, cited above). To establish the sufficiency of this resolution, additional simulations were run after dividing the branches at a higher resolution (Fig. 4D).

Soma to dendritic tip paths comprised $19 \pm 6$ compartments, ranging from 3 to 36 . Considering the number of inhibitory synapses received by TCNs (Liu et al., 1995; Sato et al., 1997) and their uniform area density (Fig. 1B), $\delta=$ $0.01 \lambda$ corresponds to one synapse per compartment on average.

All trials were run under Linux on either Pentium II, III, or AMD Athlon machines using NEURON (Hines and Carnevale, 1997) with a fixed time step of $25 \mu \mathrm{s}$.

Synaptic currents were specified by:

$I_{\mathrm{pre}}=I_{\mathrm{syn}}(t)=P \times \mathcal{W}(t)$

$I_{\text {dyn }}=I_{\text {syn }}\left(t, V_{\mathrm{m}}\right)=g_{\mathrm{syn}}(t) \times\left(V_{\mathrm{m}}-E_{\mathrm{syn}}\right)$

where $t, V_{\mathrm{m}}, E_{\text {syn }}$ are the time, membrane and reversal potentials, $P$ is the peak amplitude

$g_{\text {syn }}(t)=P \times \mathcal{W}(t)$

$\mathcal{W}(t)=c_{1} \times\left(\widehat{\mathcal{W}}\left(t+t_{\epsilon}\right)-c_{2}\right)$

$\widehat{\mathcal{W}}(t)=\mathrm{e}^{-\left(t-t_{0}\right) /\left(\tau_{\mathrm{d}} / \psi\right)}-\mathrm{e}^{-\left(t-t_{0}\right)\left(\tau_{\mathrm{r}} / \psi\right)}$

where $c_{1}, c_{2}$ and $\widehat{\mathcal{W}}$ translate and normalize $\mathcal{W}$

$\psi=\mathcal{Q}_{10}^{\left(T-21^{\circ} \mathrm{C}\right) /\left(10^{\circ} \mathrm{C}\right)}$

Low threshold T-type calcium current was defined as:

$I_{\mathrm{t}}=p_{\mathrm{Ca}} \times m^{2} h \times \mathcal{D}\left(\left[\mathrm{Ca}^{2+}\right]_{i},\left[\mathrm{Ca}^{2+}\right]_{o}, V_{\mathrm{m}}\right)$

Activation:

$\frac{\mathrm{d} m}{\mathrm{~d} t}=\frac{M_{\infty}-m}{M_{\tau} / \psi}$

$M_{\infty}=\frac{1}{1+\mathrm{e}^{\left(-V_{\mathrm{m}}+v_{1}\right) / v_{2}}}$

$M_{\tau}=s_{1}+\frac{s_{2}}{\left(\mathrm{e}^{-\left(V_{\mathrm{m}}+v_{3}\right) / v_{4}}+\mathrm{e}^{\left(V_{\mathrm{m}}+v_{5}\right) / v_{6}}\right)}$

$\psi=\mathcal{Q}_{10}^{\left(T-24^{\circ} \mathrm{C}\right) / 10^{\circ} \mathrm{C}}$

Inactivation: analogous to activation, with

$H_{\tau}=s_{1}+\frac{s_{2}}{\left(1+\mathrm{e}^{-\left(V_{\mathrm{m}}+v_{3}\right) / v_{4}}\right) /\left(1+\mathrm{e}^{\left(V_{\mathrm{m}}+v_{5}\right) / v_{6}}\right)}$

Goldman-Hodgkin-Katz drive

$\mathcal{D}\left(x_{1}, x_{2}, x_{3}\right)=x_{1} \times \mathcal{F}\left(-x_{3}\right)-x_{2} \times \mathcal{F}\left(x_{3}\right)$
$\mathcal{F}(x)=z \times F \times \frac{x}{\mathrm{e}^{\theta}-1}$

$\theta=z \times F \times \frac{V_{\mathrm{m}}}{R(T+273.15 \mathrm{~K})}$

where $z, F, R$ are the valence, gas and Faraday constants.

The constants $v_{i}$ and $s_{i}$ were determined by fitting the equations to voltage clamp data recorded in thalamocortical neurons in vitro (Coulter et al., 1989).

\begin{tabular}{llllllllll}
\hline & $v_{1}$ & $v_{2}$ & $v_{3}$ & $v_{4}$ & $v_{5}$ & $v_{6}$ & $s_{1}$ & $s_{2}$ & $\mathcal{Q}_{10}$ \\
\hline Activation & 58 & 6.2 & 133 & 17 & 18 & 18 & 1 & 1 & 2.5 \\
Inactivation & 79 & 4 & -45 & 50 & 76 & 6 & 30 & 4200 & 2.5 \\
\hline
\end{tabular}

The leak current was given by:

$I_{\mathrm{lk}}=g_{\mathrm{lk}} \times\left(V_{\mathrm{m}}-E_{\mathrm{lk}}\right)$

Under TTX and excitatory amino acid blockers APV and CNQX/DNQX, miniature inhibitory postsynaptic currents (mIPSCs) in these neurons reverse near the chloride Nernst potential and are completely abolished by $G A B A_{A}$ antagonist bicuculline (Ulrich and Huguenard, 1997; LeFeuvre et al., 1997).

$I_{\text {pre }}$ and $I_{\text {dyn }}$ were constrained using mIPSC voltage clamp recordings from rat ventrobasal TCNs slices in vitro (LeFeuvre et al., 1997; Cox et al., 1997). Peak mIPSC amplitudes are $20 \pm 1.6 \mathrm{pA}$ (Ulrich and Huguenard, 1996), $27 \pm 12 \mathrm{pA}$ (LeFeuvre et al., 1997) and $12.2 \pm 1.3 \mathrm{pA}$ (Cox et al., 1997), which convert to 420, 440, and $270 \mathrm{pS}$, respectively. The former two are raw averages, the latter is the average (across cells) of the mean of the first normal mode. Times-to-peak average $2 \pm 0.3 \mathrm{~ms}$ (LeFeuvre et al., 1997). The decay phase of averaged mIPSCs was fitted with single exponential functions, with time constants $13.9 \pm 1.4 \mathrm{~ms}$ $\left(20-22^{\circ} \mathrm{C}\right)$ (LeFeuvre et al., 1997).

Pipette solutions contained cesium, a potassium channel blocker, which approximately triples input resistance to $400 \mathrm{M} \Omega$ in rat ventrobasal TCNs in vitro (LeFeuvre et al., 1997; Zhang et al., 1997). To set the model's $R_{\text {in }}$ in this range, $g_{\mathrm{lk}}$ was set uniformly to $13 \mu \mathrm{S} / \mathrm{cm}^{2}$, which is $1 / 3$ the level obtained when fitting with the cesium-free in vitro voltage clamp recordings taken from the same cell. This yielded an input resistance of $398 \mathrm{M} \Omega(-80$ and $-85 \mathrm{mV})$ when a low threshold T-type calcium conductance was also included.

Temperature sensitivity of $G A B A_{A}$ IPSC kinetics was set at $\mathcal{Q}_{10}=2.1$, which is in agreement with the 2.0 to 2.5 range for TCNs, as well as hippocampal granule cells (Huntsman and Huguenard, 2000; Otis and Mody, 1992; Warren et al., 1994). The reversal potential of the $G A B A$-ergic chloride conductance in TCNs is $-81 \pm 2.6 \mathrm{mV}$ when the cation selective ionophore gramicidin is used to established perforated patches (Ulrich and Huguenard, 1997). 


\section{Results}

We used computer models to examine possible sources variability of miniature synaptic currents in thalamocortical neurons of the rodent ventrobasal complex. Variability was assayed by sampling the entire dendritic arbor: triggering one synapse per location and recording responses at the soma (Fig. 1A). With 1416 such compartments, this corresponded to one event every $5 \mu \mathrm{m}$ of dendritic length, on average. We compared our assays with in vitro recordings of spontaneous quantal release at $G A B A_{A}$-type synapses (Cox et al., 1997; LeFeuvre et al., 1997; Ulrich and Huguenard, 1996). Although $G A B A$-ergic currents are not always inhibitory, these events are commonly referred to as mIPSCs.

\subsection{Paradigm 1: recordings of predefined synaptic currents}

We first injected simulated miniature synaptic currents into dendritic sites and recorded the events with a somatic voltage clamp carrying $1 \mathrm{M} \Omega$ of series resistance. The injected current $I_{\text {pre }}=I_{\text {syn }}(t)$, followed a double exponential time course constrained with in vitro data at $21^{\circ} \mathrm{C}$. In analogy to "mIPSC", we call each recording an "mPRE", where "PRE" signifies the "predefined" nature of these currents. By injecting the same current at all 1416 dendritic locations, these data sets quantify the resistive-capacitive filtering of the dendrites.

\subsubsection{Synapse distributions}

Raw datasets were then weighted to account for the density of $G A B A$-ergic synapses at each location (Fig. 1B). Studies using reconstructive serial electron microscopy indicate that GABA synapses are located throughout TCN dendrites (Liu et al., 1995; Sato et al., 1997), at densities ranging from 3 to 12 synapses per $10 \mu \mathrm{m}$ of length depending inversely on distance from the soma. By factoring in dendrite diameters (Dr. Fumi Sato, personal communication), we found that the number of synapses per unit area is relatively constant (heavy lines). This is in contrast to glutamatergic synapses on TCNs, whose area densities have a strong dependence on distance (light lines).

\subsubsection{Amplitudes and rise times}

To create a physiological time course for $I_{\text {pre }}$, exponential parameters were refined iteratively until the median mPRE matched in vitro data. The fitting led to a peak amplitude of $14 \mathrm{pA}$ and to time constants $\tau_{\mathrm{r}}=0.1 \mathrm{~ms}$ and $\tau_{\mathrm{d}}=13.9 \mathrm{~ms}$. The $10-90 \%$ rise time of this waveform was $0.18 \mathrm{~ms}$, while its $0-100 \%$ rise time (time-to-peak) was $0.50 \mathrm{~ms}$. The resulting current is depicted in Fig. 2A as the dashed trace. Also depicted are somatic recordings (solid traces) of injections at the five locations indicated in Fig. 2B.

Synaptic events scattered over the entire arbor appeared in the somatic recordings with variable amplitude (Fig. 2C).
The range of peak amplitudes was 11.1-12.7 pA with median $12.3 \mathrm{pA}$. The magnitudes of these peak amplitudes indicate that somatic voltage clamps carrying $1.0 \mathrm{M} \Omega R_{\mathrm{S}}$ pick up at least $80 \%$-but no more than $90 \%$ - of any synaptic current peak regardless of location (both limits are remarkable).

The range of $10-90 \%$ rise time for the mPREs was $0.45-1.95 \mathrm{~ms}$ (median $0.98 \mathrm{~ms}$ ). Analogous in vitro statistics have not been published, although there are detailed kinetics for spontaneous IPSCs (Huntsman and Huguenard, 2000).

The diagonal in Fig. 2C relates peak amplitude to rise time under least squares constraint. The five locations that lie along the diagonal and span its length are shown as open squares in Fig. 2E. These five locations will be used latter to assess influences of various biophysical and experimental parameters. Note the uppermost open square in Fig. 2E, which marks the location that yielded the median mPRE, which is also marked by the large crosshair in Fig. 2C. This median dendritic location will be used in Paradigm 2 to constrain the conductance based model used there.

\subsubsection{Comparison to in vitro data}

A broader, $80 \mathrm{pA}$ range of variability in peak amplitudes is observed in recordings of mIPSCs in thalamocortical neurons when studied under analogous voltage clamp and $\left[\mathrm{Cl}^{-}\right]$ conditions (Fig. 2D). The modal peak of these in vitro distributions is at $12.2 \pm 1.3 \mathrm{pA}$ (Cox et al., 1997).

To show the relative breadth of mIPSC variability compared to mPRE variability, we have re-plotted in Fig. 2D the amplitude histogram of Fig. 2C. The mPRE variability gathers into a single stripe. Because we specified $I_{\text {pre }}$ as a predetermined current, local input resistance and escape voltages were irrelevant in these trials. Because we used identical $I_{\text {pre }} \mathrm{S}$, synapse heterogeneity was not an issue. In fact, resistive-capacitive filtering was the only factor giving rise to mPRE variability. We conclude that resistive-capacitive filtering represents a tiny source of synaptic variability in these neurons. For the robustness of this result, see subsequent control trials.

Note that resistive-capacitive filtering led to a larger number of large amplitude mPREs than small amplitude ones, as indicated by the skew in the amplitude histogram of Fig. 2C. In contrast, in vitro data show a preponderance of low amplitude events and such distributions have the opposite skew, as indicated in Fig. 2D. Skew will be discussed in subsequent sections as well as Section 4.

A similar mPRE/mIPSC comparison is not possible for rise times due to an absence of relevant in vitro data. However, given the approximately linear relation between rise time and amplitude (Fig. 2C), the in vitro rise time skew might be expected to be opposite to the rise time skew induced by resistive-capacitive filtering, but this is under the assumption that resistive-capacitive filtering is its primary determinant. 
(A)

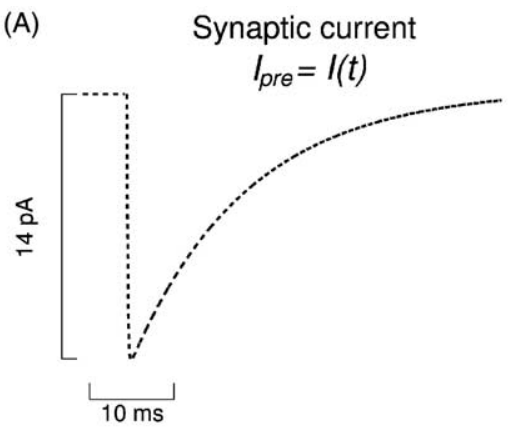

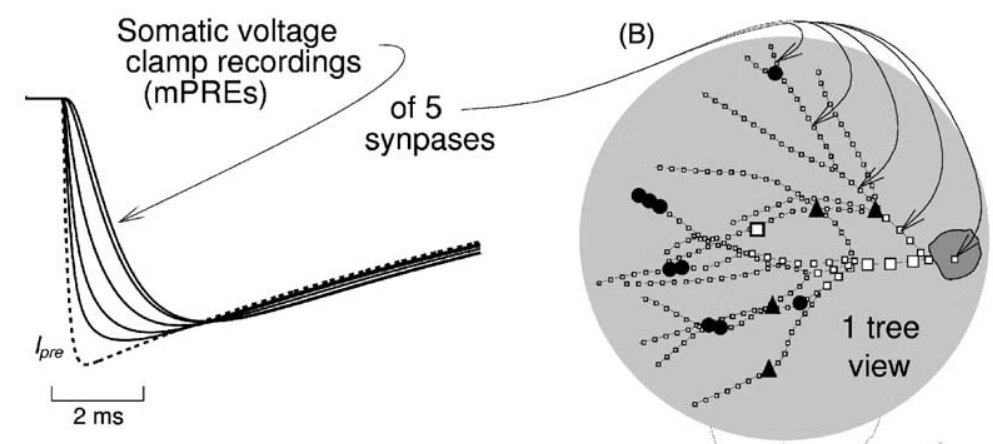

(E)
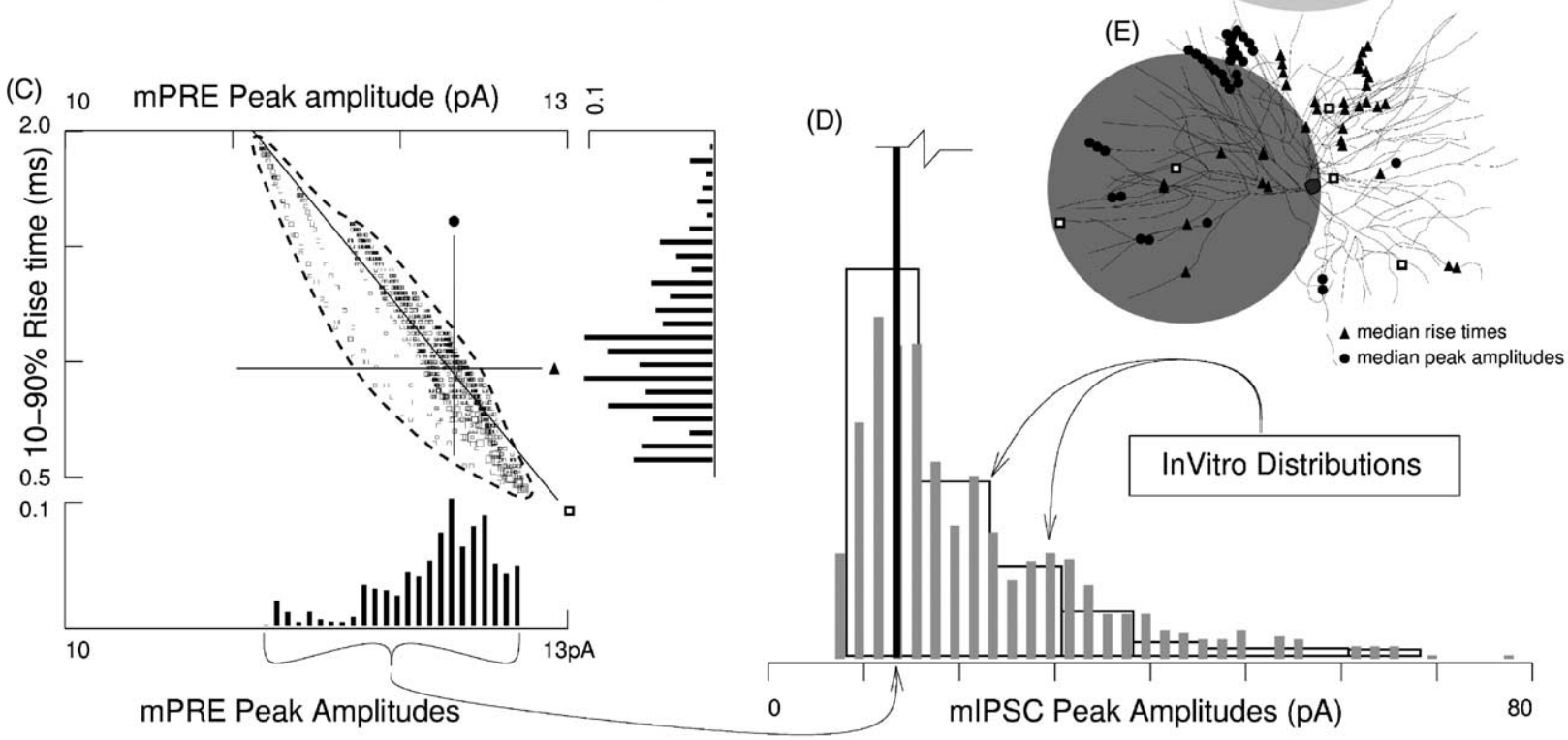

Fig. 2. Paradigm 1: synaptic variability due to resistive-capacitive filtering. Identical predetermined synaptic currents $I_{\text {pre }}=I_{\text {syn }}(t)$ were injected alternately into each of 1416 dendritic compartments. $I_{\text {pre }}$ followed a double exponential time course, which we constrained with cesium based whole cell recordings of mIPSCs in TCNs of rat ventrobasal slice preparations maintained in vitro (Cox et al., 1997; LeFeuvre et al., 1997). These dendritic injections were recorded though a somatic voltage clamp. (A) Dashed trace depicts $I_{\text {pre }}$. Solid traces depict five example somatic voltage clamp recordings (mPREs). (B) Sites of five injections recorded somatically in (A). (C) mPRE data scatter plot, with marginal histograms. Each square marks the rise time and peak amplitude of the recording of an event at one of the compartments. The large crosshair marks the median rise time and median peak amplitude, with the closest mPRE defined as the median. Even though all injected currents were identical, their recordings were variable. This variability reflects how events at different dendritic sites are subjected to different degrees of low-pass filtering when viewed from the soma. The low-pass filtering is due to three factors: (1) membrane resistance, (2) membrane capacitance, and (3) morphology of the dendritic arbor. Note that the mIPSC amplitudes have the opposite skew of the mPRE amplitudes. Also note that the range of mPRE amplitudes is less than 10\% of the range of mIPSC amplitudes. (D) Three histograms superimposed: one mPRE data set and two mIPSC data sets (Cox et al., 1997; LeFeuvre et al., 1997). Note the positive skew of mIPSC amplitude data. Each in vitro histogram is composed of several hundred events. Corrections were made for the differences in net ionic drive between the two studies; bin widths are 2 and 10pA. (E) Bold open squares mark five synapses lying on and spanning the least squares line in (C). Uppermost square marks median in (C). Triangles and circles mark locations where the injected current resulted in an mPRE lying along the lines of the crosshair.

\subsubsection{Dendritic organization}

Next, we identified all dendritic locations that yielded an mPRE rise time close to the median (i.e. along the horizontal line of Fig. 2C). There were 36 such locations within the resolution of our numerical integration. These are marked as triangles in Fig. 2E. On the other hand, the circles in Fig. 2E mark the locations that yielded the $36 \mathrm{mPRE}$ amplitudes closest to the median (i.e. along the vertical line of Fig. 2C). These two groups are patently disjoint, indicating that resistive-capacitive filtering organizes the arbor in two distinct manners: phase and attenuation yield independent parameterizations of dendritic arbors (Neubig and Destexhe, 2001).

\subsubsection{Rise times: $10-90 \%$ versus $0-100 \%$}

Due to noise, $0-100 \%$ rise times (times-to-peak) are difficult to extract from in vitro recordings; in Fig. 3 it is plotted against the $10-90 \%$ rise time. The diagonal relates the two under least squares constraint: $\Delta t_{10 / 90}=0.62 \times \Delta t_{0 / 100}-$ $0.38 \mathrm{~ms}$. For reference, the median marked by the horizontal line is $2.18 \mathrm{~ms}$ (range $1.38-3.90 \mathrm{~ms}$ ), while the median marked by the vertical line is $0.98 \mathrm{~ms}$ (range $0.45-1.95 \mathrm{~ms}$ ). 


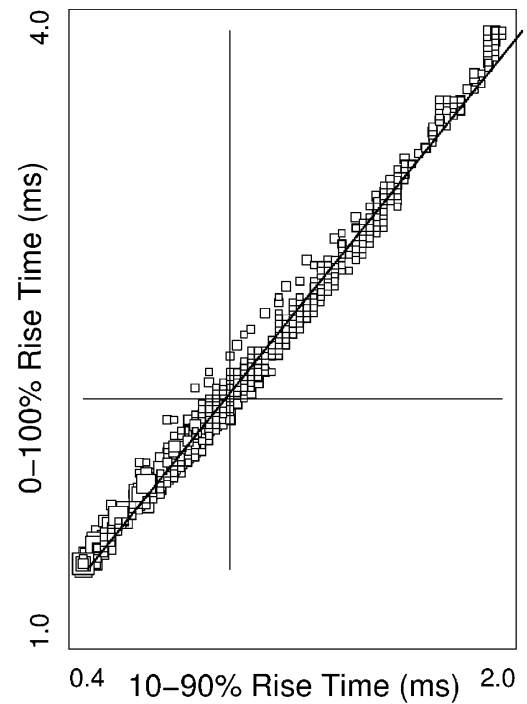

Fig. 3. Temporal measures: cross plot of two different measures of the rising phase of the somatic recordings. Each square marks the corresponding values of the recording of an event in one of the compartments. Note that the $0-100 \%$ rise time can be accurately estimated from 10 to $90 \%$ rise time. Equation of the least square line is given in text.

\subsection{Control trials: currents and parameters}

In this section, we present control trials for a number of biophysical and experimental parameters.

\subsubsection{Active membrane currents}

Thalamocortical neurons contain many voltage gated conductances, notably the low threshold calcium current $I_{\mathrm{t}}$ (Coulter et al., 1989; Crunelli et al., 1989). To verify that quantal events are too small to elicit $I_{\mathrm{t}}$, we set membrane permeability to calcium uniformly at $8 \mu \mathrm{m}^{3} / \mathrm{ms}$ (Fig. 4A). This led to a $4.7 \mathrm{nA}$ peak in the IV curve (not shown), which is midrange for intact TCNs (Destexhe et al., 1998a,b; Neubig and Destexhe, 1998a). Five trials were then run using $I_{\text {pre }}$ at the five locations specified by the bold squares in back in Fig. 2E. Recall that these locations were chosen because they yielded mPREs that spanned the full range of mPRE amplitudes and rise times. The new mPREs were nearly identical to those before-the differences being less than $0.3 \%$ in amplitude and less than $25 \mu \mathrm{s}$ in rise time. This indicates not only an absence of differential activation, but also an absence of any activation. TCNs also contain

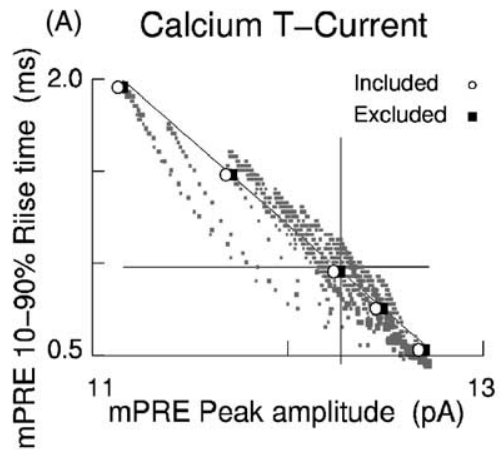

(D) Computational Resolution

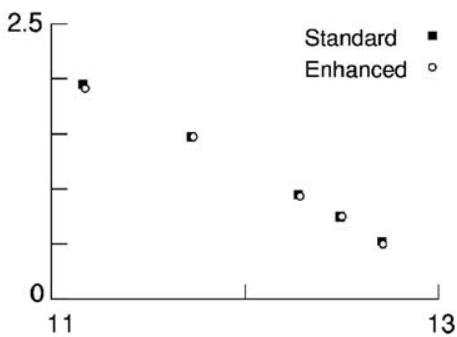

(B) Series Resistance

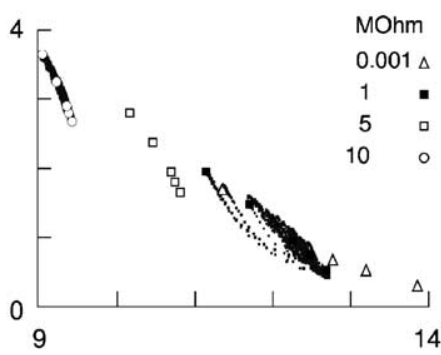

(E) Input Resistance

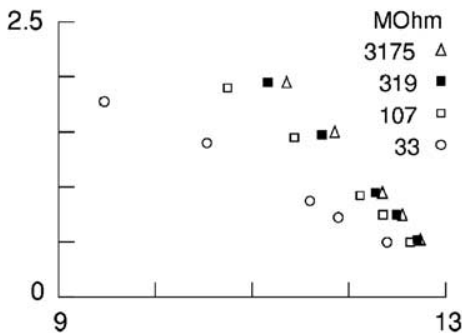

(C) Temperature

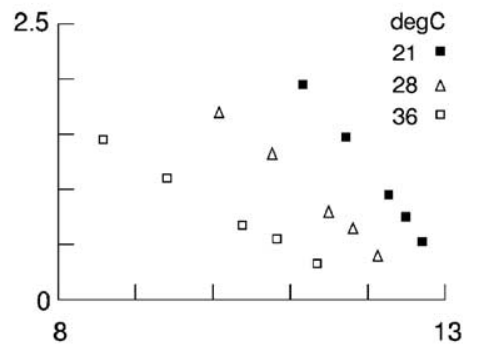

(F) Membrane Capacitance

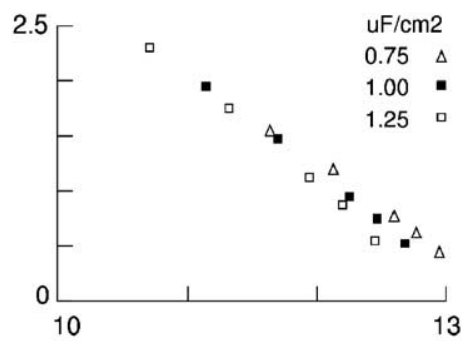

Fig. 4. Control trials: currents and parameters. Black squares indicate baseline settings in this report. (A) Inclusion of $4.7 \mathrm{nA}$ of the low threshold Ca ${ }^{2+}$ T-current led to mPREs that were slightly smaller than when excluded. All active currents, except for those carried by $\mathrm{Ca}^{2+}$, had been blocked during experiments. (B) Higher settings for the voltage clamp's series resistance led to more precise (low scatter), but less accurate (offset) recordings of synaptic

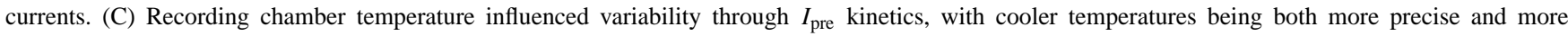
accurate. (D) Doubling the number of compartments and halving the integration time step led to minute changes, indicating that mPRE scatter (Fig. 2C) is a signature of the dendritic arbor. (E) Drastic changes in somatic $R_{\text {in }}$ led to small changes in amplitudes, suggesting that recording solutions are free to be chosen based on functions other than rendering the neuron more "compact". (F) Specific membrane capacitance influenced amplitude variability and temporal variability to roughly equal extents. (A-F) Note the preserved spatial relations (approximate co-linearity and relative inter-point distances) between the data of the five synapses. 
$I_{\mathrm{K}_{\mathrm{a}}}, I_{\mathrm{K}_{2}}, I_{\mathrm{h}}$, and $I_{\mathrm{Na}_{\mathrm{p}}}$ (Huguenard and McCormick, 1992; McCormick and Huguenard, 1992), however during in vitro recordings (Fig. 1C), they had been pharmacologically blocked.

\subsubsection{Series resistance}

We examined series resistance $\left(R_{\mathrm{S}}\right)$ by generating full data sets for $R_{\mathrm{S}}=1.0$ and $10 \mathrm{M} \Omega$ and five-point trials run at $R_{\mathrm{S}}=0.001$ and $5 \mathrm{M} \Omega$ (Fig. 3B). The broadest mPRE amplitude range, $2.5 \mathrm{pA}$, was obtained for ultra-low $R_{\mathrm{s}}$. The range diminished to $11 \%$ of $I_{\text {pre }}$ for standard $R_{\mathrm{s}}$. At $R_{\mathrm{s}}=$ $5 \mathrm{M} \Omega$, the ranges no longer overlapped and at $R_{\mathrm{S}}=10 \mathrm{M} \Omega$, the range dropped below $3 \%$ of $I_{\text {pre }}$. The progression of ranges for rise time followed the same pattern.

Under high series resistance, $R_{\mathrm{S}}=10 \mathrm{M} \Omega$, amplitudes were generally smaller-indicating that high resistance recordings are less accurate. But note that the reduction in scatter at $R_{\mathrm{S}}=10 \mathrm{M} \Omega$ indicates that higher resistances afford greater precision $\left(I_{\mathrm{pre}} \mathrm{S}\right.$ were identical). The ratio of minor to major axes of the data's bounding perimeter was 0.21 at $R_{\mathrm{S}}=1.0 \mathrm{M} \Omega$ and 0.04 at $R_{\mathrm{S}}=10 \mathrm{M} \Omega$. The ratio of the two major axes was 2.1 , and the ratio of the areas was 22.1 .

If these recordings represent measurements of the actual synaptic currents (which were identical), then the recordings should be identical as well. In a situation where a given current appears without site dependency in the recordings, variability in the recordings reflects intra-synaptic organization and not extra-synaptic processing. In this sense, uncompensated $R_{\mathrm{S}}$ recordings are preferable. Alternatively, the recordings could be interpreted as measurements of the resistive-capacitive structure of the dendritic arbor. This interpretation is valid here since $I_{\text {pres }} \mathrm{s}$ were identical. In this sense, maximally compensated $R_{\mathrm{S}}$ recordings are preferable because the scatter then represents structure.

\subsubsection{Temperature}

We examined "bath temperature" next, because this modulates $G A B A_{A}$ receptor kinetics (Fig. 3C). Using a $\mathcal{Q}_{10}$ of 2.1 (Huntsman and Huguenard, 2000; Otis and Mody, 1992; Warren et al., 1994) established faster $I_{\text {pre }}$ 's at body temperature. This led to broader variability due to low-pass properties of arbors. Room temperatures led to more precise and more accurate recordings.

\subsubsection{Discritization}

We examined the adequacy of our numerical integrations by halving the time step $(d t=12.5 \mu \mathrm{s})$ and dividing each dendritic compartment in two $(N=2833)$ (Fig. 3D). Stronger tests could have been run by dividing each compartment by $3,4,5,6, \ldots$, then comparing data of the original compartment with data of an outermost new compartment within the original compartment. The enhanced resolution led to shifts in amplitude of less than $0.1 \%$ and shifts in rise time of at most one time step compared to the standard discritization. Note that the standard discritization matches the spatial resolution of the 3D reconstruction data. It also approximates the number of inhibitory synapses per TCN (Liu et al., 1995; Sato et al., 1997).

\subsubsection{Biophysical parameters}

Lastly, we examined input resistance $\left(R_{\text {in }}\right)$ and capacitance $\left(C_{\mathrm{m}}\right)$ (Figs. 3E and 3F). Our standard $R_{\text {in }}=319 \mathrm{M} \Omega$ corresponds to in vitro recordings using cesium based solutions, as in our constraint data. A $23 \%$ increase in amplitude variability resulted when we decreased $R_{\text {in }}$ to $107 \mathrm{M} \Omega$, which corresponds to in vitro conditions with cesium-free solutions. A 93\% increase resulted when we decreased $R_{\text {in }}$ to $33 \mathrm{M} \Omega$ (white circles), which corresponds to in vivo recordings. Increasing $R_{\text {in }}$ to $3175 \mathrm{M} \Omega$ decreased the amplitude range by less than $10 \%$. These trials indicate that pharmacological manipulations of $R_{\text {in }}$ have only a small effect on mIPSC recordings in TCNs, implying that the choice of solutions is free to be based on other criteria.

Manipulations of $R_{\text {in }}$ were carried out by changing $g_{1 \mathrm{k}}$, using factors of 1/10, 3 and 9. Therefore, these trials also characterize the influence of membrane resistivity. $C_{\mathrm{m}}$, which has been shown to be relatively constant across different neuronal types (Gentet et al., 2000), influenced amplitude variability to similar extent, yet $C_{\mathrm{m}}$ 's influence on the rise time variability was much stronger.

\subsection{Paradigm 2: recordings of dynamic clamp synaptic currents}

In this section, we switch to a conductance model of miniature synaptic currents, $I_{\mathrm{syn}}\left(t, V_{\mathrm{m}}\right)=g_{\mathrm{syn}}(t) \times\left(V_{\mathrm{m}}-\right.$ $\left.E_{\text {syn }}\right)$ (Fig. 5). The analogous technique in vitro is called dynamic clamping (Sharp et al., 1992, 1993a,b), so we will refer to these currents as "I $I_{\text {dyn }}$ " and "mDYN". We use in vitro data to indirectly constrain the time course of conductance $g_{\text {syn }}(t)$, a double exponential. By imposing the same conductance time course at each location, our mDYN data sets characterize the dendritic processing that is attributable to three factors: local variations in $R_{\text {in }}$ and space clamp, and resistive-capacitive filtering. Because resistive-capacitive filtering had been the only factor shaping mPRE data, the difference between the mPRE and mDYN data sets will characterize the joint impact of $R_{\text {in }}$ and space clamp on variability of miniature synaptic currents (later we will show that $R_{\text {in }}$ is eight times more potent than space clamp quality).

\subsubsection{Amplitudes and rise times}

The uppermost open square in Fig. 2E had marked the location with the median mPRE from Paradigm 1. To constrain the time course of $g_{\mathrm{syn}}(t)$ for this paradigm, we relied on that location. With the reversal potential $E_{\text {syn }}$ set to reflect the elevated intra-cellular chloride concentration used in vitro, we refined the remaining parameters until $I_{\text {dyn }}$ at the median location yielded an mDYN that matched 
(A)

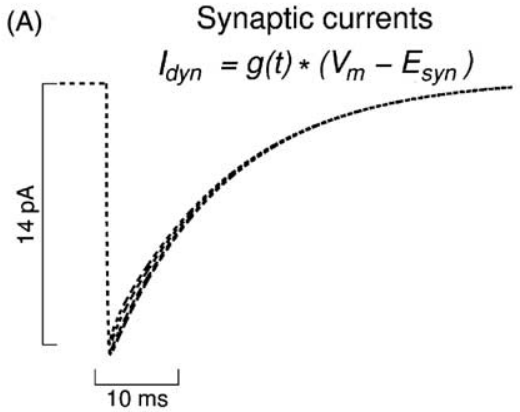

(C)

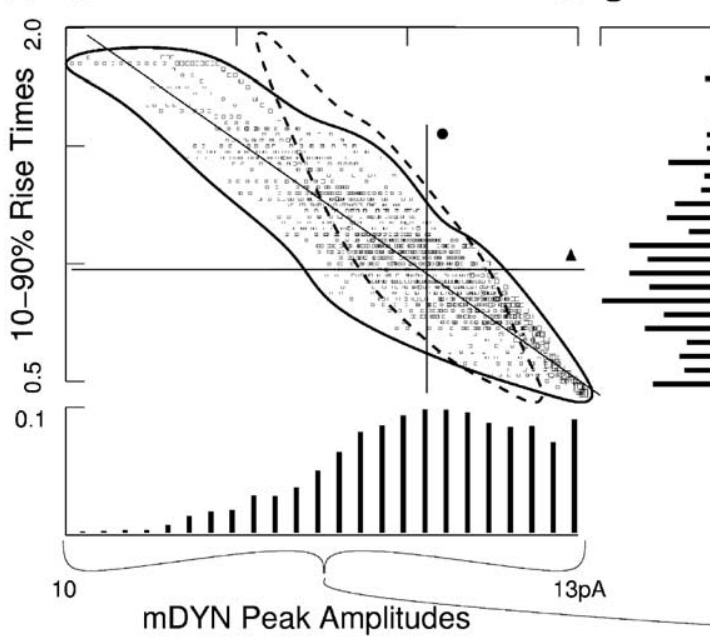

Somatic voltage clamp recordings

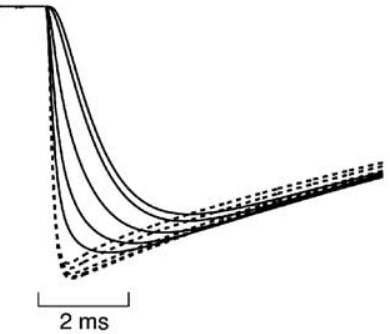

(D)
(B) Perisynaptic escape voltages

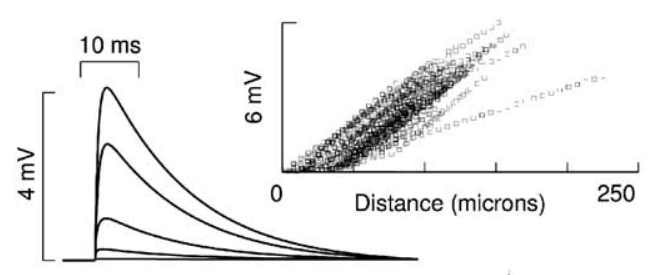

(E)

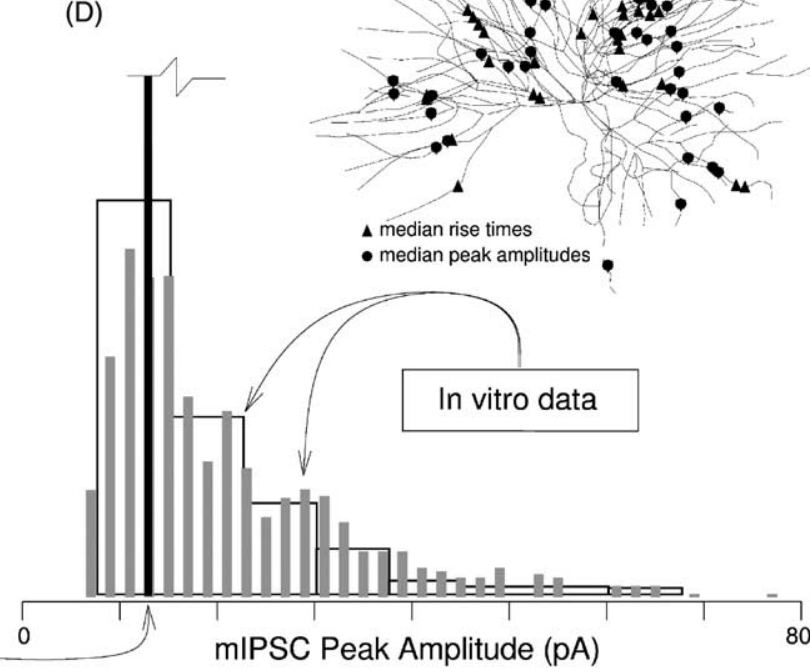

Fig. 5. Paradigm 2: synaptic variability due to filtering, input resistance, and space clamp. Conductance based synaptic currents $I_{\mathrm{dyn}}=I_{\mathrm{syn}}\left(t, V_{\mathrm{m}}\right)$ were injected alternately in each of 1416 dendritic compartments. The conductance time course $g_{\text {syn }}(t)$ was defined by a double-exponential time course constrained such that the location that had yielded the median mPRE (uppermost square in Fig. 2E) now yielded a matching mDYN. (A) Spatial variation in local $R_{\text {in }}$ and local space clamp quality led to a different $I_{\text {dyn }}$ at each location. Five example $I_{\text {dyn }} \mathrm{s}$ and mDYNs are given, corresponding to locations in Fig. 2B. Note the two-fold increase in variability of peak amplitude relative to Fig. 2A. (B) Perisynaptic escape voltages reflect space clamp quality and local $R_{\text {in }}$ (see also Fig. 9B4). (C) mDYN data scatter plot, with marginal histograms. Each square marks the rise time and peak amplitude of the recording of an event at one of the compartments. The large crosshair marks the median rise time and median peak amplitude, with the closest mDYN defined as the median. Even though the conductances used at all locations were identical, their recordings of their currents were variable. This variability reflects how conductance based events at different dendritic sites are subjected differentially to three factors: (1) local input resistance, (2) local space clamp quality, and (3) low-pass resistive-capacitive filtering when viewed from the soma. The low-pass filtering is due to three factors: (1) membrane resistance, (2) membrane capacitance, and (3) morphology of the dendritic arbor. Note that, like the mPREs, the mDYN amplitude data is negatively skewed. The dashed curved is a re-plot of the mPRE envelope in Fig. 2C. Note the two-fold increase in amplitude variability. (D) Three histograms superimposed: one mDYN data set and two mIPSC data sets (Cox et al., 1997; LeFeuvre et al., 1997). Note that the mIPSC amplitudes have the opposite skew of the mDYN amplitudes. Also note that the range of mDYN amplitudes is less than $10 \%$ of the range of mIPSC amplitudes. (E) Triangles and circles correspond to mDYNs closest to the median crosshair lines given in (C). Compare with Fig. 2E.

the corresponding mPRE, which led to these values: $P=$ $312 \mathrm{pS}, \tau_{\mathrm{d}}=0.1,13.9 \mathrm{~ms}$.

Applying this conductance time course at various locations led to synaptic currents that varied from site-to-site; examples are shown in Fig. 5A, where the five $I_{\text {dyn }} \mathrm{s}$ (dashed traces) correspond to the five dendritic locations marked in Fig. 2B. This type of synaptic variability is due to spatial variations in local $R_{\text {in }}$ and in voltage clamp quality. These factors had not been at work under the previous paradigm, where we had defined the synaptic current apriori. The somatically recorded mDYNs are depicted in Fig. 5A as (solid traces). Perisynaptic escape voltages - a term that refers to the local voltage transients that occur due to imperfect voltage/space clamp—are given in Fig. 5B. Escape voltages will also be reported in Fig. 8C4.

Synaptic $I_{\mathrm{dyn}} \mathrm{S}$ scattered over the entire arbor had peak currents varying between 13.3 and $14.3 \mathrm{pA}$, with a median of 13.9 pA. Recall that $I_{\text {pre }}$ had peaked at 14 pA (Fig. 2A). Here, distal $I_{\mathrm{dyn}} \mathrm{s}$ were smaller due to higher local $R_{\text {in }}$ and large escape voltages. These factors reduced the chemoelectric drive on the ions, and hence the their peak current. Rise times and times-to-peak of the $I_{\mathrm{dyn}} \mathrm{s}$, which ranged from 0.15 to $0.18 \mathrm{~ms}$ and 0.40 to $0.50 \mathrm{~ms}$, were uniformly less than or equal to those of $I_{\text {pre }}(0.18$ and $0.50 \mathrm{~ms})$. 
Through the somatic voltage clamp, $I_{\mathrm{dyn}} \mathrm{s}$ appeared as 10.0-13.0 pA mDYNs (Fig. 2C). This range, being 1.9 times broader than in Paradigm 1, indicates that site dependent local $R_{\text {in }}$ and site dependent space clamp quality approximately double the variability attributable to resistive-capacitive filtering. The median mDYN amplitude was $12.1 \mathrm{pA}$, whereas the median mPRE amplitude had been $12.3 \mathrm{pA}$. The median rise times and times-to-peak were identical between the two paradigms: $0.98 \mathrm{~ms}$ (range $0.45-1.95 \mathrm{~ms}$ ) and $2.18 \mathrm{~ms}$ (range $1.38-3.85 \mathrm{~ms}$ ).

\subsubsection{Comparison to in vitro data}

To show the relative breadth of mIPSC variability compared to mDYN variability, we copied onto Fig. 5D the amplitude histogram of Fig. 5C. Despite the two-fold increase in variability over Paradigm 1, the mDYN histogram collapsed to a single stripe. This indicates that together, resistive-capacitive filtering, local $R_{\text {in }}$, and local space clamp quality are tiny sources of synaptic variability in thalamocortical neurons.

As in the resistive-capacitive filtering scenario of Paradigm 1, a larger number of large amplitude mDYNs compared to small amplitude mDYNs were recorded here, even though new factors came into play ( $R_{\text {in }}$ and space clamp). This led again to a negative skew in the amplitude histogram as can be seen in Fig. 5C. In vitro data (Fig. 5D) show the opposite asymmetry. In contrast, the skew and range of variability in rise time data (Fig. 5C) was similar to the mPRE data (Fig. 2C). Analogous in vitro data have not been published, although there are detailed kinetics for spontaneous IPSCs (Huntsman and Huguenard, 2000).

\subsubsection{Dendritic organization}

We tracked the locations that yielded mDYN rise times close to the median, i.e. along the horizontal line of Fig. 5C.
These locations are marked by the 34 triangles in Fig. 5E, while the circles mark locations corresponding the to 34 mDYNs with amplitudes closest to the median, i.e. along the vertical line of Fig. 5C. As in Paradigm 1, these two sets are disjoint, indicating again that phase and attenuation constitute independent parameterizations of dendritic arbors (Neubig and Destexhe, 2001).

\subsection{4. mPREs versus $m D Y N s$}

There was a qualitative difference between the MPRE and mDYN data sets (Figs. 2C and 5C). In each scatter plot, data lie along monotonic curves. Each curve corresponds to a single dendritic path. For mPREs, the curves are concave upward, indicating rise times increase in an exponential-like manner as a function of amplitude. For mDYNs, the curves are concave downward, indicating rise times saturate as a function of amplitude.

The qualitative difference between the mPRE and $\mathrm{mDYN}$ data sets can be seen in Fig. 6, where we have cross plotted their peak amplitudes and rise times and have overlaid their maps of median locations. The first panel clearly depicts (1) the doubling of variability in the dynamic clamp paradigm, and (2) grossly different structuring of the arbor by resistive-capacitive filtering compared to local $R_{\text {in }}$ and space clamp quality. The on-diagonal scatter in the left panel reflects the parameterization of the dendritic arbor created by resistive-capacitive filtering, while the off-diagonal scatter reflects the structure created by spatial variation in $R_{\text {in }}$ (in Fig. 9B4 we show that $R_{\text {in }}$ is eight times more potent than space clamp). The relative potency of these two factors vary from branch to branch, with the structure of terminal branches being dominated by $R_{\text {in }}$, whereas resistive-capacitive filtering dominates the structure in low order branches. The difference between them can be seen again in the third panel, where the two sets of median

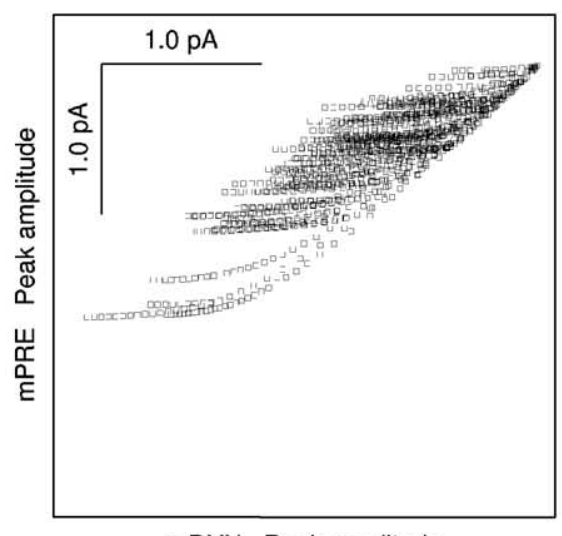

mDYN Peak amplitude
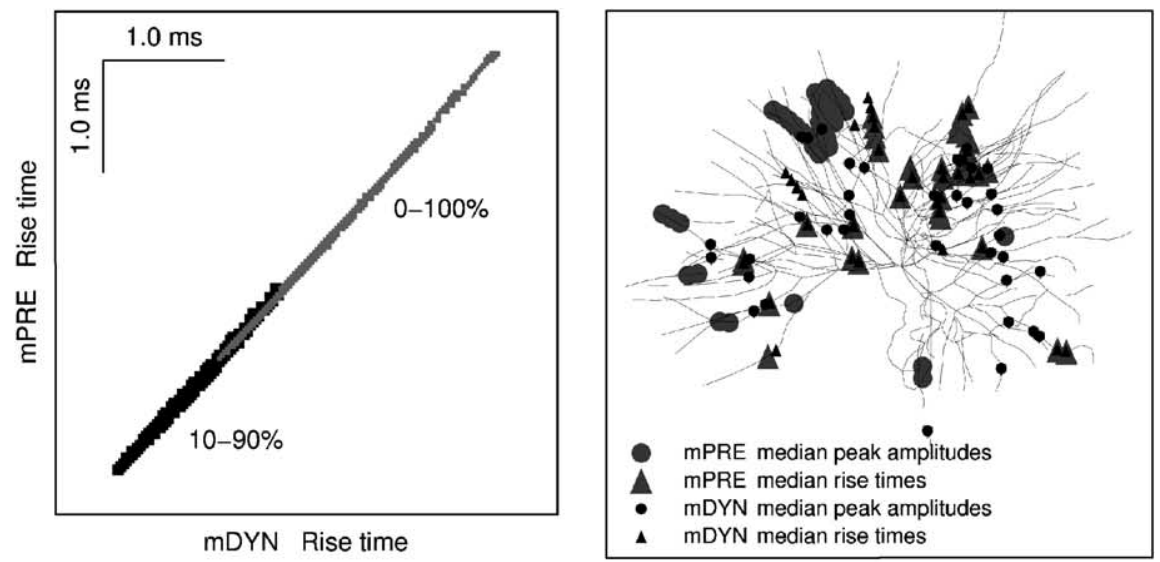

Fig. 6. Synaptic current vs. synaptic conductance. The mDYN data from the conductance based currents, $I_{\mathrm{dyn}}=I_{\mathrm{syn}}\left(t, V_{\mathrm{m}}\right)=g_{\mathrm{syn}}(t) \times\left(V_{\mathrm{m}}-E_{\mathrm{syn}}\right)$, shows twice the range of peak amplitude variability compared to mPRE data from the predetermined current modeling, $I_{\text {pre }}=I_{\text {syn }}(t)=P \times W(t)$. mDYN data is shaped by local $R_{\text {in }}$ and local space clamp quality, as well as resistive-capacitive filtering whereas only resistive-capacitive filtering shapes the mPRE data. These differences between paradigms impact peak amplitudes, as shown in the left panel, which is a cross plot of amplitude data. The differences do not impact temporal measures, as shown in the middle panel, which contains two cross plots: one for each of the indicated measures of the rising phase. The right panel depicts the superposition of Figs. 2E and 5E. 
peak amplitude locations coincide in a couple locations at most.

The temporal structure is shown in the middle panel where we have used large marks for the $10-90 \%$ rise time data so that they can be seen when behind the $0-100 \%$ rise time data. The minute amounts of off-diagonal scatter indicate that resistive-capacitive filtering (which again generates the on-diagonal scatter) dominates the temporal parameterization of the arbor compared to local $R_{\text {in }}$ and space clamp quality. This can be seen in the third panel as well, where the two sets of median rise time locations coincide in all but a few instances.

\subsection{Control trials: morphology}

We ran control trials for neuronal morphology by uniformly and non-uniformly altering the dendritic arbor. The morphology (and electrophysiology) of our reconstructed TCN is representative of rodent ventrobasal TCNs (Huguenard and Prince, 1992), whose diversity has been characterized in neuroanatomical studies (Ohara and Havton, 1994; Ohara et al., 1995). Because differences occur in overall size, not structure, the first set of alterations consisted of rescaling branch length and diameter to reflect the range of naturally occurring diversity.

Note that controlling for the range of diversity could not have been accomplished by the alternative approach of using several reconstructed neurons. A multi-reconstruction approach could not have extended results to neurons of other brain regions that have similar dendritic structure, but which fall outside the size range of TCNs. Lastly, scaling controls for systematic errors in reconstruction (staining, fixation, digitization, ... ).

\subsubsection{Rescalings}

First, we uniformly decreased dendrite diameters by a factor of 1.5 and drew a minimal envelope containing the resulting $\mathrm{mDYN}$ rise time/peak amplitude data (Fig. 7). The thinned dendrites generated more variability, increasing the range from 3 to $5 \mathrm{pA}$, but thickening dendrite diameters by the same factor led to a decrease in the variability from 3 to $1.6 \mathrm{pA}$. These relatively small changes indicate that

Fig. 7. Uniform rescaling: to extend the present results across nuclei and species we created three new models with alternative morphologies based on our reconstructed neuron and on the literature. Here, uniform rescalings included length doubling, diameter thickening and diameter thinning. These are the only ways in which rodent ventrobasal thalamocortical neurons are known to differ from each other (Ohara and Havton, 1994; Ohara et al., 1995), however we have greatly exceeded the natural range of variation in order to extend the results to other nuclei and species. Simulations were run on these new models. Associated mDYN data envelopes are plotted for each, along with marginal histograms of peak amplitudes. Range of variability in peak amplitude remained less than $10 \%$ of the range of mIPSC in vitro data. The skew of peak amplitudes continued to be opposite of MIPSC in vitro data.
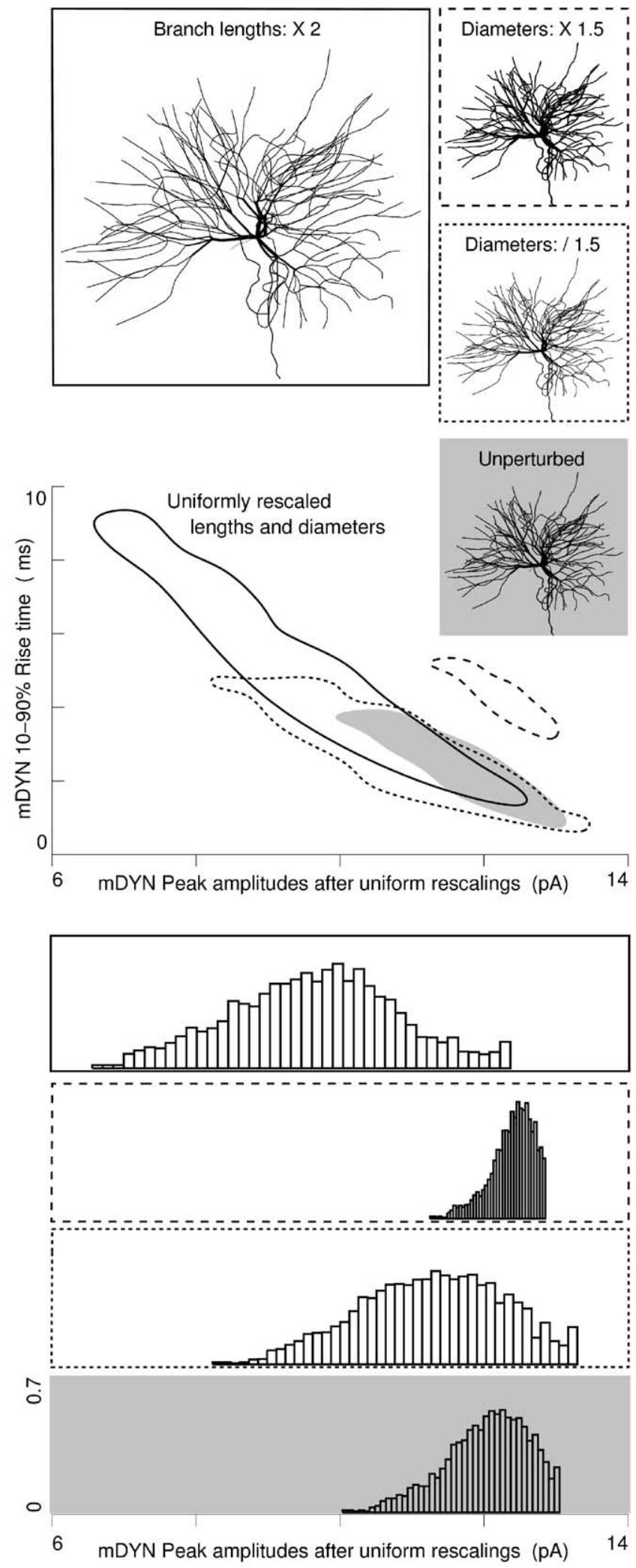
our results are robust against errors in this most difficult measurement in the 3D reconstruction process. Doubling the length of each branch increased the range of mDYN peak amplitudes from 3 to $5.7 \mathrm{pA}$. Similar changes were found in rise times (ordinates).

All three new morphologies generated a larger number of large amplitude mDYNs than small amplitude ones, as shown in the negatively skewed amplitude histograms in the lower panels. Such skew is in the opposite direction to the skew of in vitro mIPSC data sets.

\subsubsection{Branch point geometry}

Signal transmission depends heavily on dendritic branching patterns. The reconstructed TCN comprised of 179 branches connected by 52 two-descendant, 12 threedescendent, 2 four-descendent and 1 each of five-, six-, and seven-descendent branch points. Branching up to order 7 (mean and median: 4) can be seen in top panel of Fig. 8, where branch order is plotted as a function of distance from the soma. Of the 69 branch points, the median distance to the soma was $42 \mu \mathrm{m}$, as indicated by the first vertical line, which, as is characteristic of bushy arbors, is proximal to the distance that divides the net membrane area in half (67 $\mu \mathrm{m}$, second vertical line).

In the background, the lengths and positions of 108 terminal branches are sorted by remoteness. As is characteristic of bushy arbors, the median length of terminal branches was $51 \mu \mathrm{m}$ (range 5-195 $\mu \mathrm{m}$ ), which was much longer than both the $16 \mu \mathrm{m}$ median length of the other 71 branches (range 3-65 $\mu \mathrm{m})$ and the $34 \mu \mathrm{m}$ median length of all branches. Consequently, the median start distance of terminal branches was $51 \mu \mathrm{m}$.

A theoretical parameter relevant to dendritic processing is the ratio at branch points of dendrite diameters, given by $\Sigma d_{i}^{3 / 2} / D^{3 / 2}$ for daughter branch diameters $d_{i}$ and parent branch diameter $D$. Diameters of parent branches are indicated by the relative size crosses in the top panel of Fig. 8,

Fig. 8. Diameters at branch points: to further extend the present results across nuclei and species we created three more models with new alternative morphologies. Diameters were tuned to homogenize the $3 / 2$ ratios to values of the unperturbed maximum, unperturbed minimum, or unity, given by $\Sigma d_{i}^{3 / 2} / D^{3 / 2}$ for daughter branch diameters $d_{i}$ and parent branch diameter $D$. In the top panel, cross marks are placed at branch position and order and are sized to reflect $D$, revealing the characteristically rapid reduction in dendrite diameter. The 108 terminal branches are sorted by remoteness of the proximal end, stretched out horizontally and shown in grey. Second panel plots the $3 / 2$ ratio of the 69 branch points. Vertical lines mark the median branch point distance (left), and the distance marking the 50/50 split of membrane area into distal and proximal halves (right). This left/right relationship reflects the bushy nature of thalamocortical arbors. Simulations were run on these new models, and resulting mDYN data envelopes are plotted, along with histograms of peak amplitudes. Again, range of variability in peak amplitude remained less than $10 \%$ of the range of mIPSC in vitro data. Except for the morphology with $3 / 2$ ratios homogenized to the maximum, the skew of peak amplitudes continued to be opposite of MIPSC in vitro data. where the rapid reduction in branch diameter-yet another characteristic of bushy arbors-is apparent.

Given these several characteristics, the proximal to distal trend is from small $3 / 2$ ratios to large $3 / 2$ ratios, ranging from 0.4 to 2.8 with a median of 1.5 . They are plotted as a function of distance from the soma in the second panel of Fig. 8.
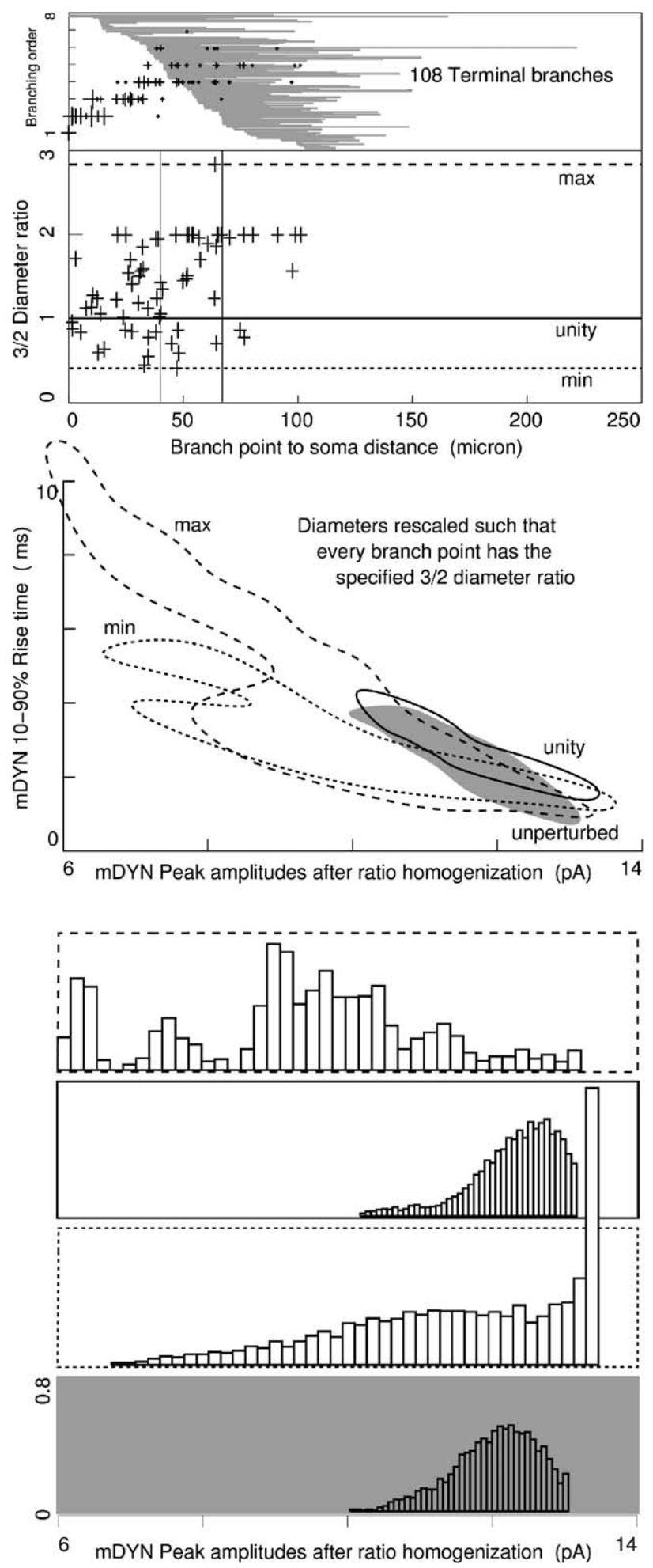
We created three new morphologies by rescaling diameters such that the $3 / 2$ ratios were identical throughout the arbor and equal to either the unperturbed maximum, unperturbed minimum, or unity as indicated by the horizontal lines in the second panel. We then uniformly rescaled the diameters to regain the initial net area, which also ensured that the average diameter per unit length did not change.

Despite the wide range of $3 / 2$ ratios in the reconstructed TCN, the new unity morphology yielded surprisingly similar mDYN peak amplitudes and rise times, as can be seen in the third panel. Interestingly, the maximum and minimum morphologies yielded different ranges of rise times but similar ranges of peak amplitudes when compared with each other.

Despite differences, all six control morphologies yielded mDYN peak amplitudes whose ranges were less than $10 \%$ of the range of in vitro data. Therefore, we conclude that our results are probably extensible to TCNs across nuclei and species: the mechanisms of resistive-capacitive filtering, local $R_{\text {in }}$, and space clamp quality play minor roles minor in synaptic variability in these cells.

\subsection{Multiple quanta and heterogeneity}

We address here two other sources of variability: multiple quanta and heterogeneous synapses.

\subsubsection{Coincident events}

A portion of the synaptic variability recorded in vitro may be due to temporal overlap of two or more quantal releases. We created such a double event dataset by reusing Paradigm 2 data. mDYNs from different locations were randomly paired, aligned by onset, then summed. Each mDYN was used exactly twice, creating 1416 of the more than 2 million possible double events. Thus we consider only maximal overlap, neglecting partial overlap and/or any facilitation/depression. Given the low frequency of minis in vitro and given the large number of $G A B A$-ergic synapses received by individual TCNs, these are not significant sources of variability.

As evident in Fig. 9A, the resulting double event amplitude range did not overlap the single event range, indicating
(A)

$$
\text { Single + double quanta events }
$$

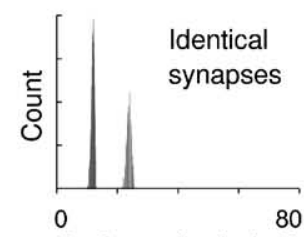

Peak amplitude (pA)

$\left(B_{2}\right)$

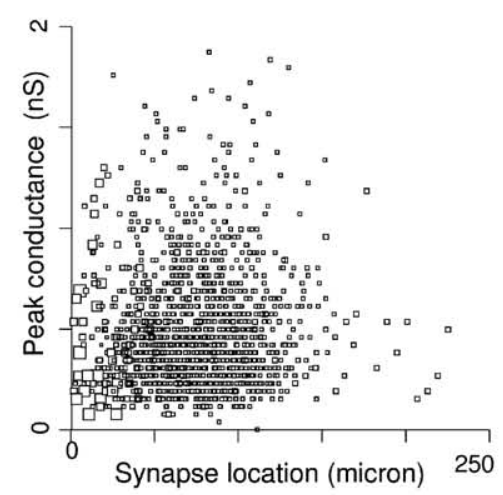

$\left(B_{1}\right)$ Single quanta events

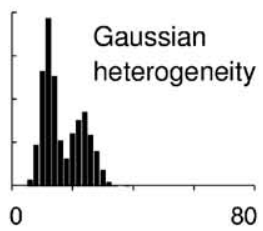

80

$\left(\mathrm{B}_{3}\right)$

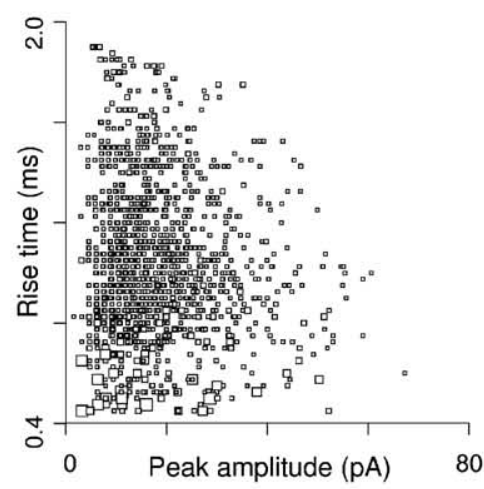

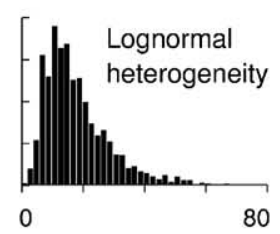

80
(C)

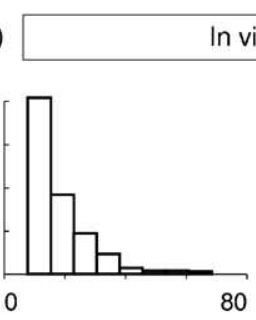

0

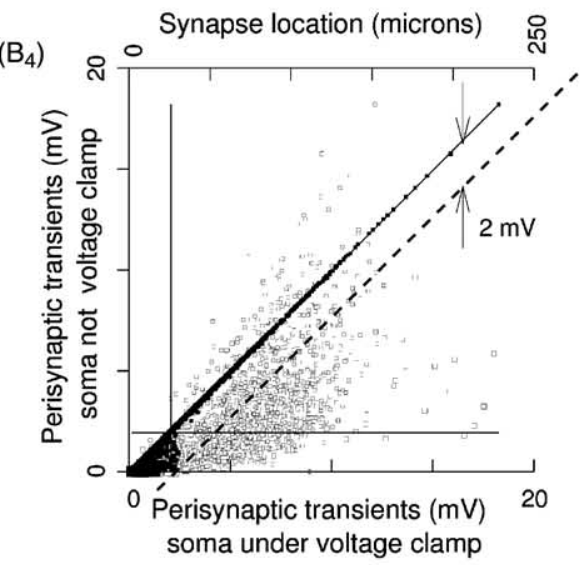

Fig. 9. Multiple events, heterogeneous conductances: multiple quantal events appear as distinct modes in amplitude histograms, even with heterogeneous synapses. In contrast, in vitro histograms are unimodal. (A) Joint histograms of single and double quantal events, for the two cases of identical and heterogeneous synapses. Heterogeneity was implemented as site dependent variation in peak conductances. Thus the histograms reflect the net heterogeneity in all parameters that shape the postsynaptic current. The heterogeneous histogram was constructed using a 10:7 ratio of single and double events so as yield a histogram similar to in vitro mIPSC data. However, this high proportion of double events is not plausible given the $4 \mathrm{~Hz}$ in vitro rate of mIPSCs. (B1) Single quantal events from heterogeneous synapses given by a lognormal distribution. Lognormal parameters were chosen to yield a distribution similar to in vitro mIPSC data. (B2) Peak conductances shown as a function (random) of distance from the soma, for the lognormal paradigm. (B3) The mDYN data under lognormal heterogeneity. (B4) Two plots: black marks are a cross plot of perisynaptic escape voltage amplitudes with and without somatic voltage clamp. Escape voltages diminish the net drive of the ions through the chloride channels $\left(V_{\mathrm{m}}-E_{\mathrm{syn}}=46 \mathrm{mV}\right)$ by up to $40 \%$. Perisynaptic transients differ between clamped and unclamped conditions by less than $2 \mathrm{mV}$, as indicated by the dashed line $y=x+2$. Since this is less than $5 \%$ of the net driving force, it indicates that local $R_{\text {in }}$ has a much greater influence than space clamp quality. Second plot consisting of grey marks show the escape voltage peaks as a function of location for the unclamped scenario. (C) In vitro mIPSC data (Cox et al., 1997; LeFeuvre et al., 1997) re-plotted from Figs. 2D and 5D. 
that alone, multiple events cannot account for in vitro variability.

\subsubsection{Gaussian peak conductances}

We have used identical synapses spread throughout the arbor in all preceding assays. Here, we heterogenize the conductances, assigning each dendritic location a conductance peak drawn randomly from a Gaussian distribution corresponding to in vitro data $(312 \pm 0.22 \mathrm{pS})($ Cox et al., 1997). Under identical conductance kinetics, $I_{\mathrm{dyn}} \mathrm{s}$ were triggered and their recordings paired as above. We then created a composite histogram, shown in Fig. 9A, by adjusting the proportion of single and double events ratio such that composite histogram resembled in vitro histograms. A 10:7 single:double ratio accomplished this.

Because the combined data spanned a contiguous range, multiple events from heterogeneous synapses could lead to an in vitro-like range of variability, potentially explaining the distribution of mIPSC peak amplitude data. But considering the low frequency of minis in vitro $(4 \mathrm{~Hz})$, the requisite high proportion of multiple events relative to single events makes this an unlikely explanation.

\subsubsection{Non-Gaussian peak conductances}

We switched to a non-Gaussian distribution of conductances, using a lognormal distribution given by $\alpha=-0.08$ and $\beta=0.6$, which yielded the $0-1.9 \mathrm{nS}$ range of peak conductances displayed in Fig. 9B2. By design, resulting mDYNs ranged from 0 to $67 \mathrm{pA}$ in peak amplitude and from 0.4 to $1.9 \mathrm{~ms}$ in rise time (Fig. 9B3), and collected into a histogram with an in vitro-like skew (Fig. 9B1).

If equivalent data had been obtained in vitro, the common procedure would have been to estimate the underlying peak conductances by dividing the recordings by the driving force, which in our assays was $V_{\text {hold }}-E_{\text {syn }}=46 \mathrm{mV}$. This procedure leads to peak conductances ranging $0-1.5 \mathrm{nS}$, which is a $21 \%$ underestimation of the actual underlying heterogeneity.

\subsubsection{Escape voltages}

Lastly, we monitored the local voltage transients at the synapse to assess the effectiveness in the distal dendrites of a somatic voltage clamp carrying $1 \mathrm{M} \Omega$ of series resistance. These escape voltages ranged up to $18 \mathrm{mV}$ in amplitude, as shown in Fig. 9B4. They depended equally on the peak conductance (not shown) and on remoteness from the soma as can be seen in light grey in the background.

With the voltage clamp removed, another round of $I_{\mathrm{dyn}} \mathrm{S}$ were triggered. In this scenario, perisynaptic voltage transients differed from the corresponding escape voltages, but only for the $50 \%$ with the lowest amplitude, as indicated by the median crosshair in Fig. 9B4. No locations differed by more than $2 \mathrm{mV}$, which is less than $5 \%$ of the $46 \mathrm{mV}$ peak driving force. For comparison, differences in local $R_{\text {in }}$ throughout the arbor led to an $18 \mathrm{mV}$ range of transient peaks, which is $\sim 40 \%$ of the maximal driving force. Thus variation in local $R_{\text {in }}$ generates eight times more variability in synaptic variability compared to space clamp quality. This underscores the previous result shown in Fig. 4E, which indicated that somatic $R_{\text {in }}$ is not a major determinant and that recording solutions are free to be chosen to produce other effects.

\section{Discussion}

We have contrasted the amplitude variability observed in vitro with our computational assays using rodent ventrobasal thalamocortical relay neurons (Fig. 1). Using identical predetermined synaptic currents, resistive-capacitive filtering led robustly to approximately $1.5 \mathrm{pA}$ of amplitude variability (Figs. 2 and 3). Using identical dynamic synaptic currents, resistive-capacitive filtering, local input resistance, and local space clamp quality led to $\sim 3$ pA of variability (Fig. 5). Under the same paradigm, variability in neurons twice this size (Fig. 7) was still an order of magnitude narrower than the in vitro range. We examined temporally overlapping events and Gaussian conductances. Neither was able to correctly expand the range, nor impart a skew that matched in vitro data. In vitro levels of variability were obtained only when synaptic conductances were non-Gaussian (Fig. 9B).

\subsection{Distribution of synapses}

Reconstructive electron microscopy studies of synapse distributions on feline TCNs have shown that length densities of inhibitory synapses decrease from proximal to distal regions by $\sim 40 \%$ (bin width $=10 \mu \mathrm{m}$ ) (Liu et al., 1995; Sato et al., 1997). Through reanalysis, we found that area density is relatively constant (Fig. 1B). It is unknown whether all of these inhibitory synapses are spontaneously active to equal extents.

Regardless of actual distribution and spontaneity, it is conservative to adopt a uniform area density of equally active synapses (as we have done here) since it leads to the broadest possible range of synaptic variability. Should studies reveal non-uniformity in rodents, then variability due to extra-synaptic factors would be more narrow than our present assessment. This would increase the strength of our basic conclusion that amplitude variability in TCNs is due to intra-synaptic organization. However, several extenuating situations are conceivable that center around the issue of skew.

\subsection{Reversed skew}

We ran single parameter control trials for morphological, biophysical and experimental variables (Fig. 3). In each, variability continued to be more than an order of magnitude narrower than in vitro variability (Fig. 1C). If multiple parameter controls had been run, joint influences may have widened the variability further. However, the baseline 
histograms (Figs. 2C and 7C) revealed a preponderance of high amplitude events, which is the reverse of the in vitro skew (Fig. 1C). The preserved spatial relations in control trial data (Fig. 3) indicate that reversed skew is preserved; thus even if joint influences multiplicatively broadened the range to in vitro proportions, the skew would be incorrect.

Theoretically, there are synapse distribution/activity patterns capable of countering the propensity towards a reversed skew. An active population comprising (i) a few percent of proximal synapses, (ii) most intermediate synapses, and (iii) no distal synapses would be an example. Uniform participation of this subpopulation would yield a correctly skewed amplitude histogram, but would require at least $50 \%$ of the inhibitory synapses to be spontaneously inactive and would result in a more narrow variability range. Nonetheless, if one assumes that influences combine multiplicatively, then such synapse patterns might yield amplitude variability on the scale of in vitro data and with the correct skew. In this event in vitro variability may not depend on intra-synaptic organization.

\subsection{Differential activation}

Because space clamp quality in intermediate and distal dendrites was degraded, large perisynaptic voltage transients were seen (Fig. 9B4). Such transients might activate voltage gated currents. Since their amplitudes were site dependent, this would result in differential activation, which would alter amplitude variability, possibly increasing it. In TCNs, candidate currents include $I_{\mathrm{K}_{\mathrm{a}}}, I_{\mathrm{K}_{2}}, I_{\mathrm{h}}$, and $I_{\mathrm{Na}_{\mathrm{p}}}$, as well as currents carried by $\mathrm{Ca}^{2+}$, notably the low threshold calcium T-current $I_{\mathrm{t}}$ (Coulter et al., 1989; Crunelli et al., 1989; Huguenard, 1996; Jahnsen and Llinás, 1984a; Llinás and Jahnsen, 1982; Huguenard and McCormick, 1992; McCormick and Huguenard, 1992).

Although plausible, differential activation is unlikely for several reasons. (1) Fundamentally different in vitro recording paradigms have led to similar variability ranges: (a) depolarizing events have been recorded with $V_{\text {hold }}=-60 \mathrm{mV}$ and a reversed $E_{\mathrm{Cl}^{-}}$(LeFeuvre et al., 1997) (Fig. 1C1), (b) hyperpolarizing events have been recorded with $V_{\text {hold }}=$ $-10 \mathrm{mV}$ and an unreversed $E_{\mathrm{Cl}^{-}}$(Cox et al., 1997) (Fig. 1C2). (2) Escape voltages were less than $18 \mathrm{mV}$ (Fig. 9B4), indicating that paradigms (a) and (b) took place in distinct voltage regimes separated by $\sim 15 \mathrm{mV}$. (3) Paradigms (a) and (b) established net driving forces of opposite sign. (4) Active potassium, sodium and mixed currents had been blocked in both paradigms. (5) The inclusion/exclusion of $I_{\mathrm{t}}$ led to less than $0.3 \%$ change due to its window component and not through its activation (Fig. 3A).

\subsection{Multiple events and heterogeneous conductances}

Temporal overlap may contribute to variability. Our results indicated that coincident events would produce well-defined modes in amplitude histograms (Fig. 9A, B). Distinct modes are not found in in vitro data (Fig. 1C). We did not examine partially overlapping events, which underestimated the range of double event amplitudes. However, in vitro event frequency is less than $4 \mathrm{~Hz}$ in TCNs (LeFeuvre et al., 1997). Since the typical event duration is less than 1/4th the period of $4 \mathrm{~Hz}$ activity, overlapping events were not frequent enough to play a significant role.

This assumes that behaviors are not far from average. In order for non-average behavior to be significant, it would need to occur much more frequently. This could occur if the inter-event interval data for individual cells were non-Gaussian to an extent that modal values differed from the mean. This has not been reported (Cox et al., 1997; LeFeuvre et al., 1997; Leresche, 1992; Ulrich and Huguenard, 1996; Zhang et al., 1997).

We considered multiple events in combination with Gaussian conductances. Based on the mean and variance of in vitro data (Cox et al., 1997) quantal events had a contiguous range of amplitudes (Fig. 9A). However, multiple events would need to be $70 \%$ as frequent as single events to yield the correct skew. If we assume independence among spontaneous events, such a ratio is not feasible given the low frequency of events in in vitro data.

The assumption of independence deserves discussion since multiple vesicle releases would be masked if their timing were coordinated to provide simultaneous releases. In this case, the $4 \mathrm{~Hz}$ rate could not be used to argue against a large proportion of multiple simultaneous events.

One potential coordination mechanism would be the existence of multiple synapses per afferent neuron. Since a single thalamic reticular neurons forms many synapses on each target TCN, action potentials initiating spontaneously nearly anywhere in the axon arbor could coordinate releases at a number of synapses. In the present analysis, this mechanism is ruled by the bath application of TTX during recordings.

A second potential mechanism would be the disregulation in the presynaptic bouton of intra-cellular calcium concentrations. This mechanism would be a factor at boutons that support multiple release sites. While multiple release sites cannot be ruled out, disruption of calcium regulation would likely be catastrophic given the numerous cascades initiated by elevated concentrations.

\subsection{Conclusion}

Having examined voltage activated currents, resistivecapacitive filtering, local input resistance, space clamp quality, temporally overlapping events, and Gaussian heterogeneities, our overall finding is that none of these factors, either alone or in aggregate, is able to explain the distribution of quantal event amplitudes that are observed in vitro. We obtained the width, skew and unimodal nature of in vitro data only when we switched to non-Gaussian distributions of peak conductance (Fig. 9B). Our trials with 
altered morphologies and biophysical parameters extended these findings to thalamocortical general.

The rodent somatosensory system has important features that will be advantageous in future studies of synaptic variability. First, inhibition in the ventrobasal complex derives from a single type of $G A B A$-ergic neuron, which comprise the thalamic reticularis nucleus. They are its only neuronal type (Benson et al., 1992; Harris et al., 1987; Houser et al., 1980; Ohara and Lieberman, 1993; Williams and Faull, 1987). Second, depending on the animal's behavioral state, reticulothalamic inhibition serves one of two dramatically different functions. During drowsiness and non-REM sleep, inhibitory inputs to thalamocortical neurons provide phasic hyperpolarizations which initiate the rebound low threshold spikes that are essential to sleep oscillations. During wakefulness, the inhibition is part of the processing of sensory information as it passes through the thalamus before reaching the neocortex. Compared to other systems, the two distinct roles place twice the constraint on the functional role that intra-synaptic heterogeneity may play in networks. The rodent somatosensory thalamus is thus a prime candidate for molecular genetic approaches of intra-synaptic heterogeneity and of heterogeneity's role in brain function at higher levels of CNS organization.

\section{Acknowledgements}

The authors wish to thank Dr. F. Sato for providing dendrite diameter data from their electron microscopy work and discussions; Drs. N. Leresche and C. Cox for e-mail discussion. This work was supported by grants from the Howard Huges Medical Institute, the National Institutes of Health, Medical Research Council of Canada Grant. Address for reprint requests: M. Neubig, Salk CNL, 10010 N Torrey Pines, La Jolla, CA 92037.

\section{References}

Andersen, P., Eccles, J.C., 1962. Inhibitory phasing of neuronal discharge. Nature 196, 645-647.

Andersen, P., Sears, T.A., 1964. The role of inhibition in phasing of spontaneous thalamo-cortical discharge. J. Physiol. (London) 173, 459480.

Benson, D.L., Isackson, P.J., Gall, C.M., Jones, E.G., 1992. Contrasting patterns in the localization of glutamic acid decarboxylase and $\mathrm{Ca}^{2+}$ calmodulin protein kinase gene expression in the rat central nervous system. Neuroscience 46 (4), 825-849.

Brown, T.H., Wong, R.K., Prince, D.A., 1979. Spontaneous miniature synaptic potentials in hippocampal neurons. Brain Res. 177 (1), 194199.

Coulter, D.A., Huguenard, J.R., Prince, D.A., 1989. Calcium currents in rat thalamocortical relay neurones: kinetic properties of the transient, low-threshold current. J. Physiol. (London) 414, 587-604

Cox, C.L., Huguenard, J.R., Prince, D.A., 1997. Nucleus reticularis neurons mediate diverse inhibitory effects in the thalamus. Proc. Natl. Acad. Sci. U.S.A. 94, 8854-8859.
Crunelli, V., Lightowler, S., Pollard, P.E., 1989. A T-type $\mathrm{Ca}^{2+}$ current underlies low-threshold $\mathrm{Ca}^{2+}$ potentials in cells of the cat and rat lateral geniculate nucleus. J. Physiol. (London) 413, 543-561.

Destexhe, A., Bal, T., McCormick, D.A., Sejnowski, T.J., 1996. Ionic mechanisms underlying synchronized oscillations and propagating waves in a model of ferret thalamic slices. J. Neurophysiol. 76 (3), $169-185$.

Destexhe, A., Contreras, D., Steriade, M., 1998a. Mechanisms underlying the synchronizing action of corticothalamic feedback through inhibition of thalamic relay cells. J. Neurophysiol. 79, 999-1016.

Destexhe, A., Neubig, M., Ulrich, D., Huguenard, J., 1998b. Dendritic low-threshold calcium currents in thalamic relay cells. J. Neurosci. 18 (10), 3574-3588.

Edwards, F.A., Konnerth, A., Sakmann, B., 1990. Quantal analysis of inhibitory synaptic transmission in the dentate gyrus of rat hippocampal slices: a patch-clamp study. J. Physiol. (London) 430, 213-249.

Faber, D.S., Young, W.S., Legendre, P., Korn, H., 1992. Intrinsic quantal variability due to stochastic properties of receptor-transmitter interactions. Science 258, 1494-1498.

Frerking, M., Borges, S., Wilson, M., 1995. Variation in GABA mini amplitude is the consequence of variation in transmitter concentration. Neuron 15, 885-895.

Frerking, M., Borges, S., Wilson, M., 1997. Are some minis multiquantal. J. Neurophysiol. 78, 1293-1304.

Funke, K., Eysel, U.T., 1998. Inverse correlation of firing patterns of single topographically matched perigeniculate neurons and cat dorsal lateral geniculate relay cells. Vis. Neurosci. 15 (4), 711-729.

Gentet, L.J., Stuart, G.J., Clements, J.D., 2000. Direct measurement of specific membrane capacitance in neurons. Biophys. J. 79, 314-320.

Harris, R.M., Hendrickson, A.E., 1987. Local circuit neurons in the rat ventrobasal thalamus-a $G A B A$ immunocytochemical study. Neuroscience 21 (1), 229-236.

Hicks, T.P., Metherate, R., Landry, P., Dykes, R.W., 1986. Bicucullineinduced alterations of response properties in functionally identified ven troposterior thalamic neurones. Exp. Brain Res. 63, 248-264.

Hines, M.L., Carnevale, N.T., 1997. The NEURON simulation environment. Neural Comput. 9, 1179-1209.

Houser, C.R., Vaughn, J.E., Barber, R.P., Roberts, E., 1980. Gaba neurons are the major cell type of the nucleus reticularis thalami. Brain Res. 200, 341-354.

Huguenard, J.R., 1996. Low-threshold calcium currents in central nervous system neurons. Ann. Rev. Physiol. 58, 329-348.

Huguenard, J.R., McCormick, D.A., 1992. Simulation of the currents involved in rhythmic oscillations in thalamic relay neurons. J. Neurophysiol. 68 (4), 1373-1383.

Huguenard, J.R., Prince, D.A., 1992. A novel T-type current underlies prolonged $\mathrm{Ca}^{2+}$ dependent burst firing in $G A B A$-ergic neurons of rat thalamic reticular nucleus. J. Neurosci. 12, 3804-3817.

Huntsman, M.M., Huguenard, J.R., 2000. Nucleus-specific differences in $G A B A_{A}$ receptor mediated inhibition are enhanced during thalamic development. J. Neurophysiol. 83, 350-358.

Jahnsen, H., Llinás, R.R., 1984a. Electrophysiological properties of guineapig thalamic neurons: an in vitro study. J. Physiol. (London) 349,205226

Jahnsen, H., Llinás, R.R., 1984b. Ionic basis for the electroresponsiveness and oscillatory properties of guinea-pig thalamic neurones in vitro. J. Physiol. (London) 349, 227-247.

Jones, E.G., 1991. The anatomy of sensory relay functions in the thalamus. Prog. Brain Res. 87, 29-52.

Lee, S.M., Ebner, F.F., 1992. Induction of high frequency activity in the somatic sensory thalamus of rats in vivo results in long-term potentiation of responses in SI cortex. Exp. Brain Res. 90, 253-261.

Lee, S.M., Friedberg, M.H., Ebner, F.F., 1994. The role of GABA-mediated inhibition in the rat ventral posterior medial thalamus. I. Assessment of receptive field changes following thalamic reticular nucleus lesions. J. Neurophysiol. 71, 1702-1715. 
LeFeuvre, Y., Fricker, D., Leresche, N., 1997. $G A B A_{A}$-receptor mediated IPSCs in rat thalamic sensory nuclei: patterns of discharge and tonic modulation by $G A B A_{B}$ autoreceptors. J. Physiol. (London) 502, 91104.

Leresche, N., 1992. Synaptic currents in thalamocortical neurons of the rat lateral geniculate nucleus. Eur. J. Neurosci. 4, 595-602.

Liu, X.B., Honda, C.N., Jones, E.G., 1995. Distribution of four types of synapse on physiologically identified relay neurons in the ventroposterior thalamic nucleus of the cat. J. Comp. Neurol. 352, 69-91.

Llinás, R.R., Jahnsen, H., 1982. Electrophysiology of mammalian thalamic neurons in vitro. Nature 297, 406-408.

McCormick, D.A., 1992. Neurotransmitter actions in the thalamus and cerebral cortex and their role in neuromodulation of thalamocortical activity. Prog. Neurobiol. 39, 337-388.

McCormick, D.A., Huguenard, J.R., 1992. A model of the electrophysiological properties of thalamocortical relay neurons. J. Neurophysiol. 68 (4), 1384-1400.

Neubig, M., 1999. Computational analyses of patterns at the subcellular scale, of the electrophysiology of thalamocortical relay neurons. Ph.D. Thesis, Faculté de Medecine, Université Laval, Québec.

Neubig, M., Destexhe, A., 1997. Constraining morphological and biophysical parameters via minimization along behavioral metrics: low order compartmental models of thalamic relay neurons which preserve afferent synapse distributions. Soc. Neurosci. Abstr. 23, 230.3.

Neubig, M., Destexhe, A., 1998a. Dendritic calcium currents in thalamic relay cells. In: Bower, J.M. (Ed.), Computational Neuroscience Trends in Research. Plenum, New York, pp. 233-238.

Neubig, M., Destexhe, A., 1998b. LTS bursting and the subcellular localizations of T-type $\mathrm{Ca}^{2+}$ channels in thalamic relay neurons. Soc. Neurosci. Abstr. 24, 407.10.

Neubig, M., Destexhe, A., 1999. Low threshold calcium T-current IV curve geometry is alterable through the distribution of T-channels in thalamic relay neurons. Neurocomputing 26-27, 215-221.

Neubig, M., Destexhe, A., 2000. Are the conductances of inhibitory synapses on thalamic relay neurons inhomogeneous? Are synapses from individual afferents clustered? Neurocomputing 32-33, 213-218.

Neubig, M., Destexhe, A., 2001. Dendritic organization of thalamocortical relay neurons and dual functions of inhibitory synaptic drives. Thalamus 1 (1), 39-52.

Nusser, Z., Cull-Candy, S., Farrant, M., 1997. Differences in synaptic $G A B A_{A}$-receptor number underlie variation in $G A B A$ mini amplitude. Neuron 19, 697-709.

Nusser, Z., Hajos, N., Somogyi, P., Mody, I., 1998. Increased number of synaptic $G A B A_{A}$ receptors underlies potentiation at hippocampal inhibitory synapses. Nature $395,172-177$.

Ohara, P.T., Havton, L.A., 1994. Dendritic architecture of rat somatosensory thalamocortical projection neurons. J. Comp. Neurol. 341, 159-171.

Ohara, P.T., Lieberman, A.R., 1993. Some aspects of the synaptic circuitry underlying inhibition in the ventrobasal thalamus. J. Neurocytol. 22, 815-825.

Ohara, P.T., Ralston, H.J., Havton, L.A., 1995. Architecture of individual dendrites from intracellularly labeled thalamocortical projection neurons in the ventral posterolateral and ventral posteromedial nuclei of cat. J. Comp. Neurol. 358, 563-572.

Otis, T.S., Mody, I., 1992. Modulation of decay kinetics and frequency of $G A B A_{A}$ receptor-mediated spontaneous inhibitory postsynaptic currents in hippocampal neurons. Neurosci. 49 (1), 13-32.

Rall, W., 1967. Distinguishing theoretical synaptic potentials computed for different soma-dendritic distributions of synaptic input. J. Neurophysiol. 30, 1138-1168.

Rall, W., 1977. Core conductor theory and cable properties of neurons. In: APS (Ed.), Handbook of Physiology, The Nervous System. Cellular Biology of Neurons, vol. 1. American Physiological Society, Bethesda, Maryland, pp. 39-97.
Ropert, N., Miles, R., Korn, H., 1990. Characteristics of miniature inhibitory postsynaptic currents in CA1 pyramidal neurones of rat hip pocampus. J. Physiol. (London) 428, 707-722.

Salt, T.E., 1989. Gamma-aminobutyric acid and afferent inhibition in the cat and rat ventrobasal thalamus. Neuroscience 28, 17-26.

Sato, F., Nakamura, Y., Shinoda, Y., 1997. Serial electron microscopic reconstruction of axon terminals on physiologically identified thalamcortical neurons in the cat ventral lateral nucleus. J. Comp. Neurol. 388, 6133-6631.

Sharp, A.A., Abbott, L.F., Marder, E.F., 1992. Artificial electrical synapses in oscillatory networks. J. Neurophysiol. 67 (6), 1691-1694.

Sharp, A.A., O’Neil, M.B., Abbott, L.F., Marder, E.F., 1993a. The dynamic clamp: artificial conductances in biological neurons. Trends Neurosci. 16 (10), 389-394.

Sharp, A.A., O'Neil, M.B., Abbott, L.F., Marder, E.F., 1993 b. Dynamic clamp: computer-generated conductances in real neurons. J. Neurophysiol. 69 (3), 992-995.

Silver, R.A., Traynelis, S.F., Cull-Candy, S.G., 1992. Rapid-time-course miniature and evoked excitatory currents at cerebellar synapses in situ. Nature 355, 163-166.

Smetters, D.K., Nelson, S.B., 1994. Role of electrotonic structure in generating synaptic variability in cortical neurons. Soc. Neurosci. Abstr. 24, 301.10.

Smetters, D.K., Nelson, S.B., 1995. Electrotonic structure and synaptic variability in cortical neurons. In: Bower, J.M. (Ed.), Proceedings of the Third Annual Computation and Neural Systems Conference on the Neurobiology of Computation. Kluwer, Boston.

Sohal, V.S., Huguenard, J.R., 1998. Long-range connections synchronize rather than spread intrathalamic oscillations: computational modeling and in vitro electrophysiology. J. Neurophysiol. 80, 1736-1751.

Sohal, V.S., Huntsman, M.M., Huguenard, J.R., 2000. Reciprocal inhibitory connections regulate the spatiotemporal properties of intratha lamic oscillations. J. Neurosci. 20 (5), 1735-1745.

Spruston, N., Jaffe, D.B., Johnston, D., 1994. Dendritic attenuation of synaptic potentials and currents: the role of passive membrane properties. Trends Neurosci. 17 (4), 161-166.

Steriade, M., McCormick, D.A., Sejnowski, T.J., 1993. Thalamocortical oscillations in the sleeping and aroused brain. Science 262, 679685.

Stevens, C.F., 1993. Quantal release of neurotransmitter and long-term potentiation. Neuron 10 (Suppl.), 55-63.

Stuart, G., Spruston, N., 1998. Determinants of voltage attenuation in neocortical pyramidal neuron dendrites. J. Neurosci. 18 (10), 35013510 .

Turrigiano, G.G., Leslie, K.R., Desai, N.S., Rutherford, L.C., Nelson, S.B., 1998. Activity-dependent scaling of quantal amplitude in neocortical neurons. Nature 391 (6670), 892-896.

Ulrich, D., Huguenard, J.R., 1996. $G A B A_{B}$-receptor mediated responses in $G A B A$-ergic projection neurones of rat nucleus reticularis thalami in vitro. J. Physiol. (London) 493, 845-854.

Ulrich, D., Huguenard, J.R., 1997. Nucleus-specific chloride homeostasis in rat thalamus. J. Neurosci. 17 (7), 2348-2354.

Walmsley, B., Alvarez, F.J., Fyffe, R.E., 1998. Diversity of structure and function at mammalian central synapses. Trends Neurosci. 21 (2), 8188.

Warren, R.A., Agmon, A., Jones, E.G., 1994. Oscillatory synaptic interactions between ventroposterior and reticular neurons in mouse thalamus in vitro. J. Neurophysiol. 72 (4), 1993-2003.

Williams, M.N., Faull, R.L.M., 1987. The distribution and morphology of identified thalamocortical projection neurons and glial cells with reference to the question of interneurons in the ventrolateral nucleus of the rat thalamus. Neuroscience 21, 767-780.

Worgotter, F., Suder, K., Zhao, Y., Kerscher, N., Eysel, U.T., Funke, K., 1998. State-dependent receptive field restructuring in the visual cortex. Nature 396 (6707), 165-168.

Zhang, S.J., Huguenard, J.R., Prince, D.A., 1997. $G A B A_{A}$-receptor mediated $\mathrm{Cl}^{-}$currents in rat thalamic reticular and relay neurons. J. Neurophysiol. 78, 2280-2286. 